\section{Recapitulating bone development through engineered mesenchymal condensations and mechanical cues for tissue regeneration}

\author{
Anna M. McDermott ${ }^{1,2,3 *}$, Samuel Herberg ${ }^{4 *}{ }^{\dagger}$, Devon E. Mason ${ }^{1,2}$, Joseph M. Collins ${ }^{1,2,5}$, \\ Hope B. Pearson ${ }^{2}$, James H. Dawahare ${ }^{2}$, Rui Tang ${ }^{4}$, Amit N. Patwa ${ }^{6}$, Mark W. Grinstaff ${ }^{6}$, \\ Daniel J. Kelly ${ }^{3}$, Eben Alsberg ${ }^{4,7,8 \neq \S}$, Joel D. Boerckel ${ }^{1,2,5 \S}$
}

Large bone defects cannot form a callus and exhibit high complication rates even with the best treatment strategies available. Tissue engineering approaches often use scaffolds designed to match the properties of mature bone. However, natural fracture healing is most efficient when it recapitulates development, forming bone via a cartilage intermediate (endochondral ossification). Because mechanical forces are critical for proper endochondral bone development and fracture repair, we hypothesized that recapitulating developmental mechanical forces would be essential for large bone defect regeneration in rats. Here, we engineered mesenchymal condensations that mimic the cellular organization and lineage progression of the early limb bud in response to local transforming growth factor- $\beta 1$ presentation from incorporated gelatin microspheres. We then controlled mechanical loading in vivo by dynamically tuning fixator compliance. Mechanical loading enhanced mesenchymal condensationinduced endochondral bone formation in vivo, restoring functional bone properties when load initiation was delayed to week 4 after defect formation. Live cell transplantation produced zonal human cartilage and primary spongiosa mimetic of the native growth plate, whereas condensation devitalization before transplantation abrogated bone formation. Mechanical loading induced regeneration comparable to high-dose bone morphogenetic protein-2 delivery, but without heterotopic bone formation and with order-of-magnitude greater mechanosensitivity. In vitro, mechanical loading promoted chondrogenesis and up-regulated pericellular matrix deposition and angiogenic gene expression. In vivo, mechanical loading regulated cartilage formation and neovascular invasion, dependent on load timing. This study establishes mechanical cues as key regulators of endochondral bone defect regeneration and provides a paradigm for recapitulating developmental programs for tissue engineering.

\section{INTRODUCTION}

Bone fractures are among the most common traumatic injuries, but regularly heal efficiently (90 to $95 \%$ success rate) by forming a stabilizing cartilage callus that remodels to bone, a process called endochondral ossification (1). Conversely, critically sized bone defects (exceeding $3 \mathrm{~cm}$ ) cannot form a callus, do not heal without surgical intervention, and often result in life-long disability for the patient. The clinical gold standard for repairing large bone defects is currently autograft bone transplantation from the iliac crest, but it is limited by the amount of source material available and donor site morbidity (2). To overcome these limitations, recombinant human

\footnotetext{
Department of Orthopaedic Surgery, Perelman School of Medicine, University of Pennsylvania, Philadelphia, PA 19104, USA. ${ }^{2}$ Department of Aerospace and Mechanical Engineering, University of Notre Dame, Notre Dame, IN 46556, USA. ${ }^{3}$ Department of Mechanical Engineering, Trinity Center for Bioengineering, Trinity College Dublin, Dublin D02 PN40, Ireland. ${ }^{4}$ Department of Biomedical Engineering, Case Western Reserve University, Cleveland, OH 44106, USA. ${ }^{5}$ Department of Bioengineering, University of Pennsylvania, Philadelphia, PA 19104, USA. ${ }^{6}$ Department of Biomedical Engineering, Boston University, Boston, MA 02215, USA. ${ }^{7}$ Department of Orthopaedic Surgery, Case Western Reserve University, Cleveland, OH 44106, USA. ${ }^{8}$ National Center for Regenerative Medicine, Division of General Medical Sciences, Case Western Reserve University, Cleveland, OH 44106, USA.

*These authors contributed equally to this work.

†Present address: Departments of Ophthalmology, Cell and Developmental Biology, and Biochemistry and Molecular Biology, SUNY Upstate Medical University, Syracuse, NY 13210, USA.

$\ddagger$ Present address: Departments of Bioengineering and Orthopaedics, University of Illinois, Chicago, IL 60607, USA.

§Corresponding author. Email: ealsberg@uic.edu (E.A.); boerckel@pennmedicine. upenn.edu (J.D.B.)
}

bone morphogenetic protein-2 (BMP-2) is administered via collagen sponge, but large doses of this growth factor can cause harmful heterotopic bone formation and soft tissue inflammation, and its continued clinical use is under re-evaluation (3). Despite these treatment options, long-term outcomes exhibit high rates of failure and complications, and many patients report that their quality of life after such interventions is no better than amputation (4). Thus, there remains a need for alternative graft materials that require little or easily obtainable donor material, remain localized to the defect region, and successfully vascularize and integrate for functional regeneration of large bone defects.

A common tissue engineering approach to this problem features osteoprogenitor cell and/or osteoinductive agent delivery using scaffolds designed to mimic the structure and properties of mature bone tissue (5). However, natural bone healing achieves highly efficient functional repair by reactivating developmental programs, producing new bone through endochondral ossification $(6,7)$. Here, we sought to recapitulate the cellular, morphogenic, and mechanical cues present during bone development for regeneration of large bone defects.

Long bone morphogenesis is initiated by condensation of mesenchymal cells in the early limb bud, which differentiate and mature into the cartilaginous anlage that gives rise to endochondral bone formation. This process is dependent on both local morphogen gradients and mechanical forces in utero $(7,8)$. Natural bone fracture healing recapitulates this developmental program but only under mechanical conditions of axial interfragmentary motion (i.e., cyclic compression at the fracture site) (9-11). In the absence of interfragmentary strain, fractures will heal through direct intramembranous 
bone formation; conversely, excessive motion or instability can induce nonunion (12). Thus, mechanical cues are critical regulators of endochondral ossification. The emerging paradigm of biomimetic bone tissue engineering aims to replicate the endochondral process $(13,14)$, but functional endochondral bone regeneration using transplanted human progenitor cells remains elusive, potentially due to insufficient recapitulation of the essential cellular, biochemical, and mechanical stimuli.

Here, we evaluated the effects of in vivo mechanical loading on endochondral bone regeneration mediated by engineered mesenchymal condensations with local morphogen presentation. Selfassembled, scaffold-free human bone marrow-derived mesenchymal condensations containing transforming growth factor- $\beta 1$ (TGF- $\beta 1$ )releasing gelatin microspheres $(\mathrm{GMs})(15,16)$ were transplanted into critical-sized bone defects in rat femora, and in vivo mechanical loading was controlled by dynamic modulation of fixation plate compliance $(17,18)$. Mechanical loading enhanced mesenchymal condensation-induced endochondral bone formation. Mechanistically, loading regulated chondrogenesis and pericellular matrix (PCM) formation and controlled cartilage persistence and neovascularization in vivo, dependent on load timing. Together, these data demonstrate the importance of mechanical cues for mimicking development and natural repair for tissue engineering.

\section{RESULTS}

\section{Engineered mesenchymal condensations}

Human bone marrow stromal cells, also termed human mesenchymal stem cells (hMSCs), were self-assembled into sheets containing local GM-mediated presentation of TGF- $\beta 1$ (Fig. $1 \mathrm{~A}$, left), which were then formed into engineered mesenchymal condensations with cylindrical shape for in vivo implantation (Fig. 1A, right) $(19,20)$. In this study, hMSC sheets were cultured for 2 days before either in vitro analysis or mesenchymal condensation assembly and in vivo transplantation into critical-sized $(8 \mathrm{~mm})$ bone defects (Fig. 1B). Bone defects were surgically created in femora of athymic Rowett nude (RNU) rats, as described previously $(18,21)$. This 8 - $\mathrm{mm}$ segmental defect model is a challenging test bed for regenerative strategies, being $60 \%$ larger than the minimum gap size necessary to prevent spontaneous repair $(5 \mathrm{~mm})(21,22)$.

First, to verify that local TGF- $\beta 1$ presentation induced chondrogenic lineage commitment, we evaluated condensations with empty or TGF- $\beta 1$-containing microspheres at days 2 and 23 of in vitro culture in standard chondro-inductive medium (Fig. 1C). Subsequently, condensations assembled after 2 days of culture were transplanted in vivo for evaluation of bone regeneration, neovascularization, and endochondral ossification over 12 weeks (Fig. 1D). At day 2, the condensations exhibited homogeneous cellular organization without histologically detectable sulfated glycosaminoglycan (sGAG) deposition or bone formation; by day 23 , characteristically shaped chondrocytes and substantial sGAG matrix deposition were apparent (Fig. 1E and fig. S1). Local TGF- $\beta 1$ presentation up-regulated and sustained mRNA expression of Sry-box 9 (SOX9), aggrecan (ACAN), and collagen 2a1 (COL2A1) (Fig. 1F), with minimal expression of osteogenic markers [Runt-related transcription factor 2 (RUNX2), alkaline phosphatase $(A L P)$, or collagen 1a1 (COL1a1)]. Osterix (OSX) expression was up-regulated by TGF- $\beta 1$ at day 2 but suppressed in differentiated chondrocytes at day 23 (Fig. 1F). TGF- $\beta 1$ also increased phosphorylation of the chondrogenic transcription factor
SMAD3 at day 2 in vitro (Fig. 1, G and $H$ ). These data demonstrate chondrogenic lineage priming consistent with the known dynamics of TGF- $\beta 1-S M A D$ signaling and downstream gene expression in the early developing limb (embryonic days 11.5 to 12.5 ) (23-25). TGF- $\beta 1$ presentation transiently up-regulated transcription of the yes-associated protein (YAP) target gene cysteine-rich angiogenic inducer 61 (CYR61) (Fig. 1F) and increased YAP content at day 2 (Fig. 1I, top row). However, TGF- $\beta 1$-induced chondrogenic lineage commitment by day 23 abrogated YAP expression and reduced matrix, but not intracellular, immunolocalization of CYR61 (Fig. 1, F and I, bottom row).

\section{Mechanical regulation of bone regeneration in vivo}

Mechanical stimuli promote proper endochondral ossification during both bone development and fracture healing $(6,7,26)$, but the effects of in vivo mechanical loading on transplanted cell-mediated bone repair are unknown. Here, using a critical-sized rat bone defect model, we modulated ambulatory load transfer using internal fixation plates of variable stiffness. The plates allow axial deformation after unlocking (Fig. 2A and fig. S2) $(18,27,28)$. The timing and magnitude of mechanical forces imparted to the defects were controlled in three groups: stiff (control, $n=11$ ); early loading (compliant plates unlocked at implantation to allow immediate loading, $n=9$ ); and delayed loading (compliant plates unlocked to initiate loading at week $4, n=9$ ) (Fig. 2B and movie S1). The multimodal mechanical behavior of the plates was assessed by ex vivo mechanical testing (stiff: $k_{\text {axial }}=260 \pm 28 \mathrm{~N} / \mathrm{mm}$, locked compliant: $k_{\text {axial }}=250 \pm$ $35 \mathrm{~N} / \mathrm{mm}$, and unlocked compliant: $k_{\text {axial }}=8.0 \pm 3.5 \mathrm{~N} / \mathrm{mm}$; mean \pm SD; fig. S2). Published data on femoral loading during the rat gait cycle (29) and rule-of-mixtures theory were used to estimate load sharing between the fixation plates and the defect tissue. These calculations indicated that interfragmentary strains at day 0 reached 2 to $3 \%$ in the stiff and delayed groups and up to 10 to $15 \%$ in the early group. A recent in vivo strain sensor study using a modified version of the stiff plates described here confirmed these numbers within $1 \%$ for the stiff group (30). The amount of strain induced over time is a function of the load sharing and therefore dependent on the amount, composition, and kinetics of tissue ingrowth; however, accounting for load sharing by ingrowing bone, we estimated strains of 5 to $10 \%$ upon plate unlocking at week 4 in the delayed group, with all groups converging on 0.5 to $3 \%$ by week 12 .

Bone regeneration progressed through endochondral ossification, exhibiting zonal cartilage and woven bone mimetic of the native growth plate by week 4 (Fig. 2, C and D). Both early and delayed loading significantly enhanced bone formation $(P<0.05$; Fig. 2 , figs. S3 and S4, and movie S1) as measured by bone volume (Fig. $2 \mathrm{E}$ ) and bone volume fraction (fig. S3). Loading significantly elevated bone accumulation rate between weeks 4 and $8(P<0.05$; fig. S3). This coincided with the timing of load initiation in the delayed group and the tissue differentiation stage, namely, chondrocyte hypertrophy and endochondral transition, in all three groups (figs. S5 and S6). Although early loading increased bone formation (Fig. 2, E and F), the bone volume fraction response in this group was highly variable (fig. S3) and exhibited a significantly lower bridging rate compared to delayed loading ( 2 of 8 versus 6 of $8 ; P<0.05, \chi^{2}$ test; fig. S3) due to persistent regions of nonmineralized cartilage (Fig. 2, C, D, and F; and figs. S5 and S6), similar to the pseudarthroses induced by large deformation cyclic bending (31). In contrast, delayed loading induced robust bone formation (Fig. 2, E and F), with a consistent 

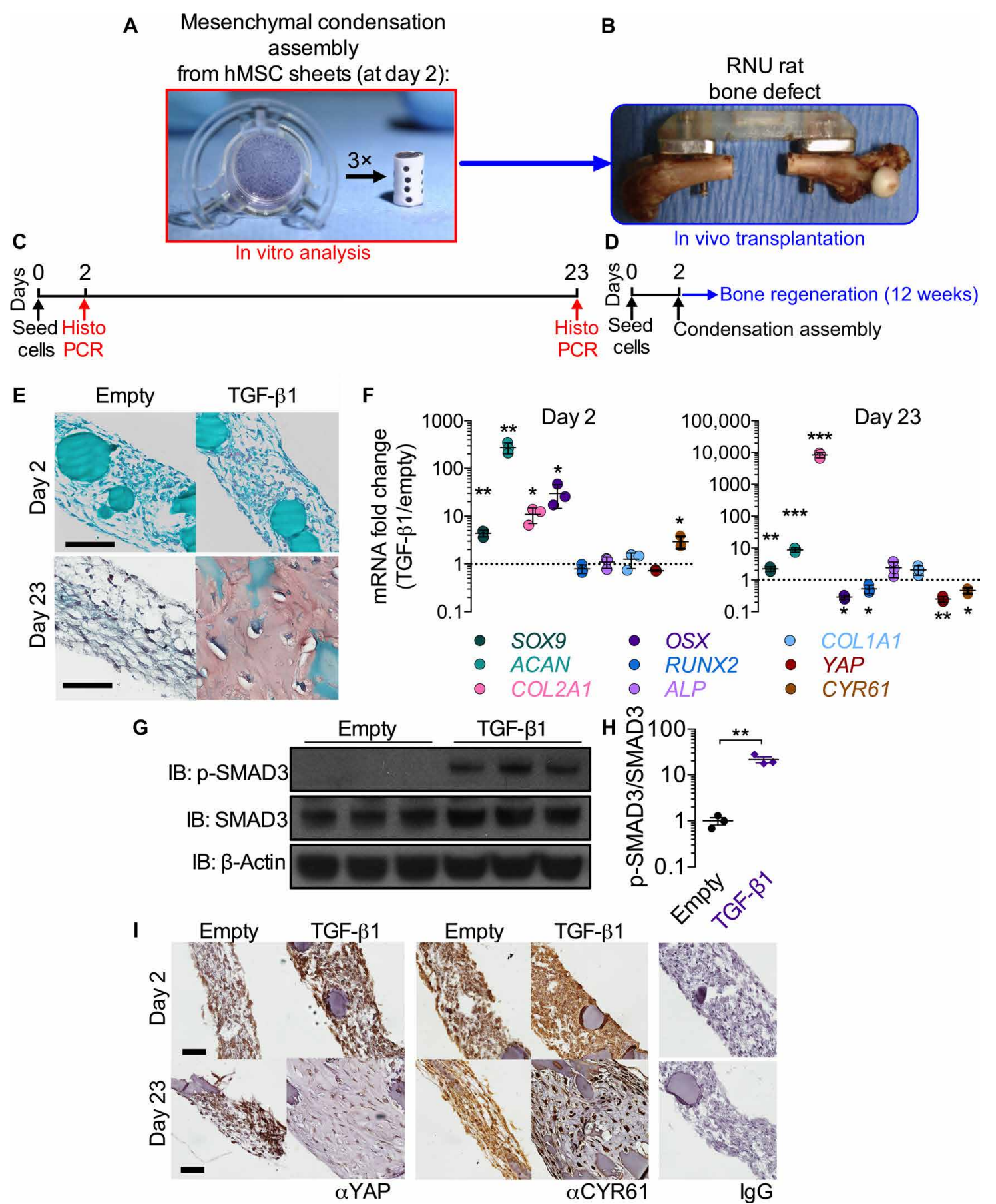

- ALP
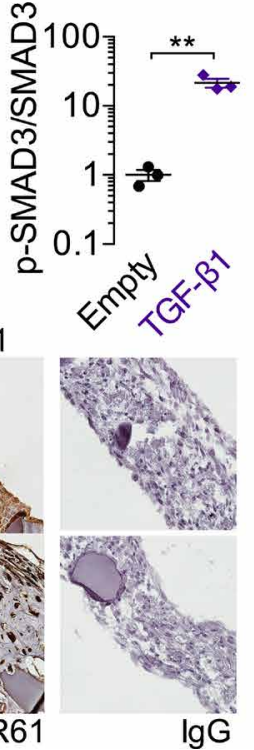

Fig. 1. Engineered mesenchymal condensations. (A) Photograph of hMSC sheets containing TGF- $\beta 1$-loaded GMs self-assembled for 2 days on Transwell inserts (left) before combination into engineered mesenchymal condensations for implantation (right). Each condensation was assembled from three sheets and enclosed within a perforated electrospun nanofiber mesh tube of polycaprolactone. (B) Photograph of a critically sized $(8 \mathrm{~mm})$ segmental bone defect created in the femora of an RNU rat. Engineered mesenchymal condensations were implanted into bone defects. (C and D) Timelines of in vitro and in vivo analyses. (C) hMSC sheets were evaluated in vitro at 2 days (the time point of transplantation) or at 23 days of culture in chondrogenesis-supportive medium. (D) Bone regeneration, neovascularization, and endochondral ossification were evaluated over 3 to 12 weeks after transplantation. (E) Safranin O/fast green staining of hMSC sheets with empty or 600 ng of TGF- $\beta 1$-containing GMs, cultured for 2 or 23 days in vitro. Red indicates sGAGs; fast green counterstain shows cells and remaining matrix. (F) mRNA expression of chondrogenic, osteogenic, and YAP pathway genes at days 2 and 23 in vitro; quantitative reverse transcription polymerase chain reaction (qRT-PCR) results normalized to glyceraldehyde-phosphate dehydrogenase (GAPDH) and expressed as fold change over empty microsphere control sheets ( $n=3$ sheets per group). (G) Immunoblot (IB) of phosphorylated-SMAD3 activity at day 2 in vitro with $\beta$-actin control and (H) band intensity of p-SMAD3/SMAD3 ratio expressed as fold change over sheets without growth factor. (I) Immunostaining for YAP and CYR61 at days 2 and 23 in vitro. 3,3'-diaminobenzidine (DAB) staining with peroxidase was used to produce a brown reaction product at locations of immunolabeled antigens. Right: Negative control isotype lgG (rabbit, top; mouse, bottom). ${ }^{*} P<0.05,{ }^{* *} P<0.01,{ }^{* * *} P<0.001$ TGF- $\beta 1$-treated versus empty, unpaired two-tailed Student's $t$ test for each independent gene. Data shown are means \pm SD. Scale bars, $100 \mu \mathrm{m}$. 
Fig. 2. Mechanical loading enhanced endochondral bone regeneration. (A) Schematic of fixation plate configurations for dynamic control of ambulatory load transfer. (B) Schematic of loading timeline. Early loading features compliant plate actuation at implantation; delayed loading features unlocking at week 4. (C) Representative in vivo microCT reconstructions at week 4. (D) Safranin $\mathrm{O} /$ fast green staining of sagittal histological sections at week 4 (left) in comparison to the native rat distal femur growth plate (right). Bottom row: Magnification of boxed areas. (E) Longitudinal microCT quantification of bone volume at week 4 $[n=11,11,9$, and 8 for stiff, early, delayed, and BMP-2/collagen (stiff), respectively], week $8(n=10$, 9,8 , and 8), and week $12(n=10,8,8$, and 8). Repeated significance indicator letters $(a, b$, and $c)$ signify $P>0.05$ (not significant); and groups with distinct indicators (a versus b) signify $P<0.05$ at each time point. (F) Representative 3D microCT reconstructions at week 12. (G) Local trabecular thickness mapping on transverse sections, indicated by boxed arrows in (F), in comparison to the native bone of the ipsilateral femoral head (H). (I) MicroCT quantification of trabecular thickness (Tb.Th), number (Tb.N), and spacing (Tb.Sp) in reference to that of the ipsilateral femoral head (femoral head mean \pm SD shown as dotted lines and shaded pink region). (J) Hematoxylin and eosin (H\&E)-stained histological sections at week 4 (representative sample from $n=1$ per group). (K) Representative 3D microCT reconstruction of BMP-1/collagen group at week 12. (L) Trabecular thickness mapping on the section indicated in (K) illustrating heterotopic bone. Scale bars, $100 \mu \mathrm{m}$. Data shown with mean \pm SEM. ${ }^{* *} P<0.01$, one- or two-way ANOVA with Tukey's post hoc analysis.

bridging rate (fig. S3). Region of interest (ROI) analyses (fig. S4) and quantitative densitometry (fig. S4) revealed mineral concentration at the defect periphery, indicative of a cortical shell, in all three groups. The bone formed within this cortex exhibited welldefined trabecular architecture, which was quantitatively similar to native femoral head trabecular bone as assessed by microcomputed tomography (microCT) morphometry (Fig. 2, G to I, and fig. S4) and histology (Fig. 2J and figs. S5 and S6).

As a clinically relevant positive control, a healing dose of recombinant human BMP-2 (rhBMP-2; $5 \mu \mathrm{g}$ per defect) (3), delivered on absorbable collagen sponge with stiff fixation, was also evaluated $(n=8)$ ("BMP-2/collagen," dashed line in Fig. 2E). This treatment produced rapid bone accumulation until week 4 and reduced bone formation rate thereafter (Fig. 2E). As reported clinically, BMP-2 treatment induced extensive heterotopic bone formation (Fig. 2, K and L). Surgeries

A

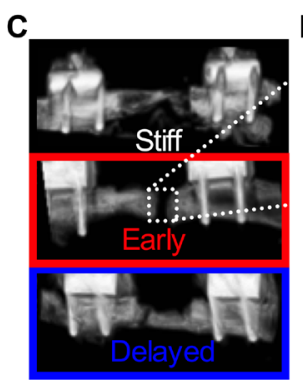

$\mathbf{F}$

G
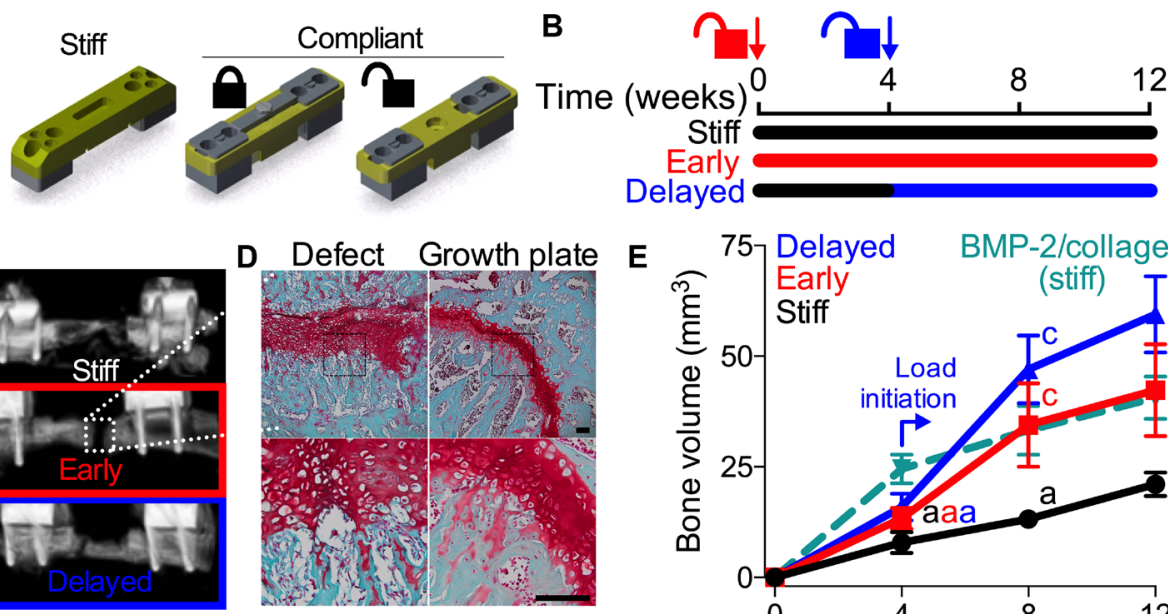

E
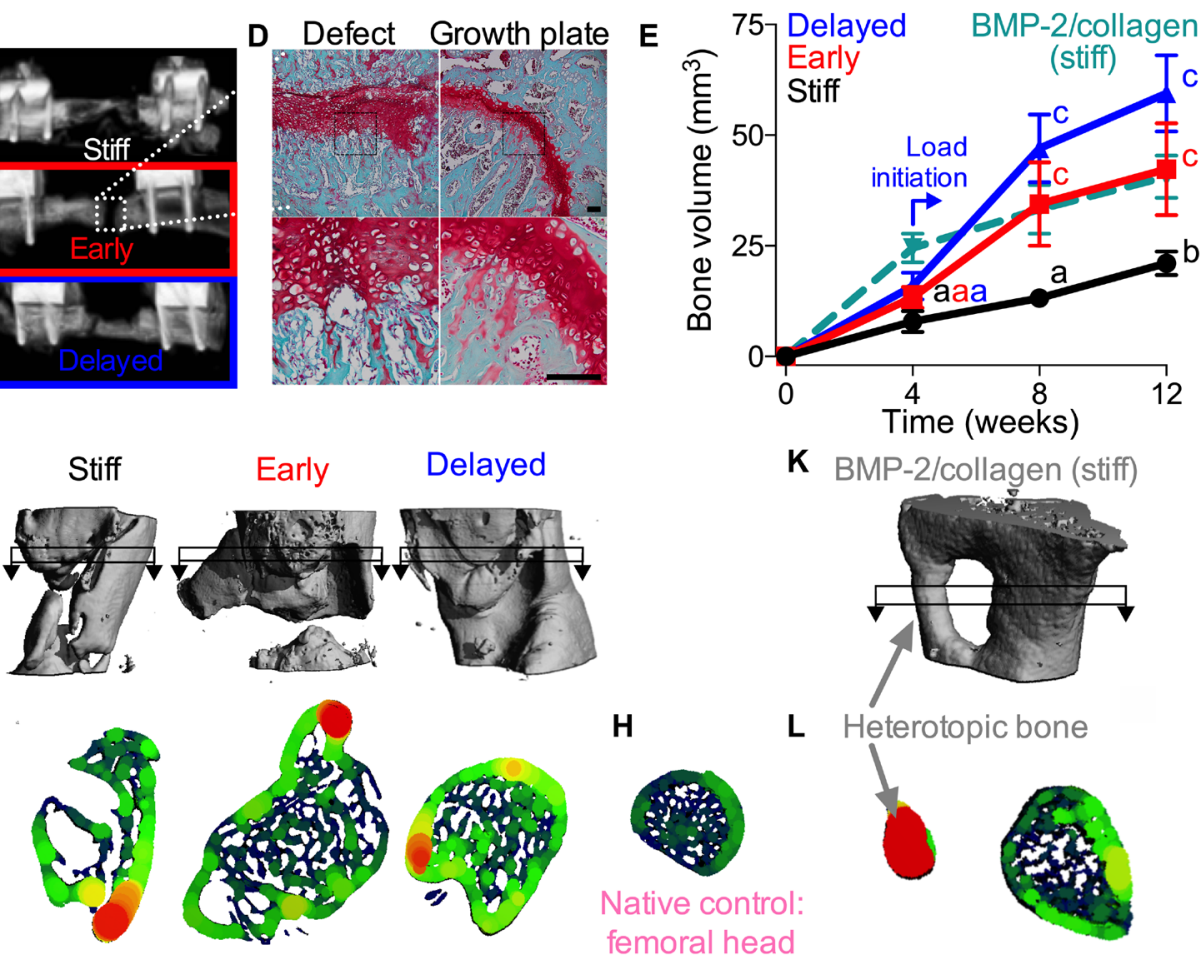

H

L Heterotopic bone

K BMP-2/collagen (stiff)

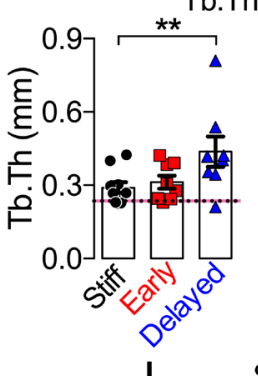

0.85

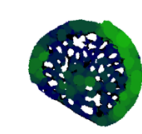

Native control: femoral head
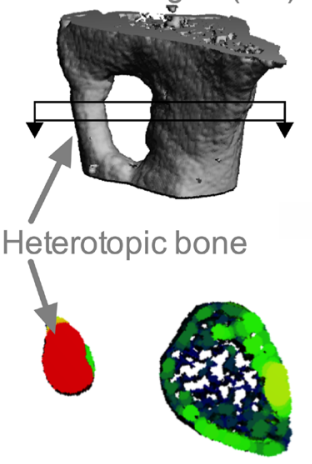

b.Th $(\mathrm{mm})$

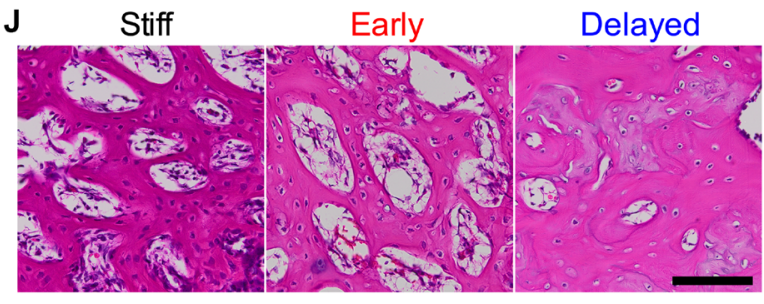

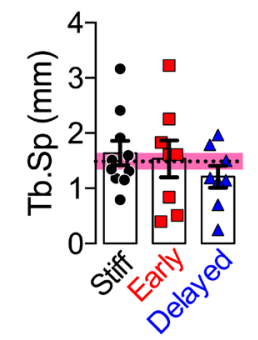

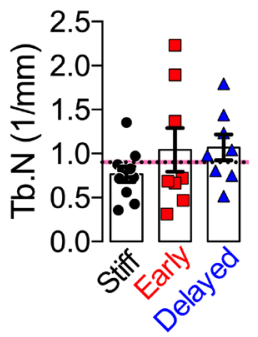

Delayed 

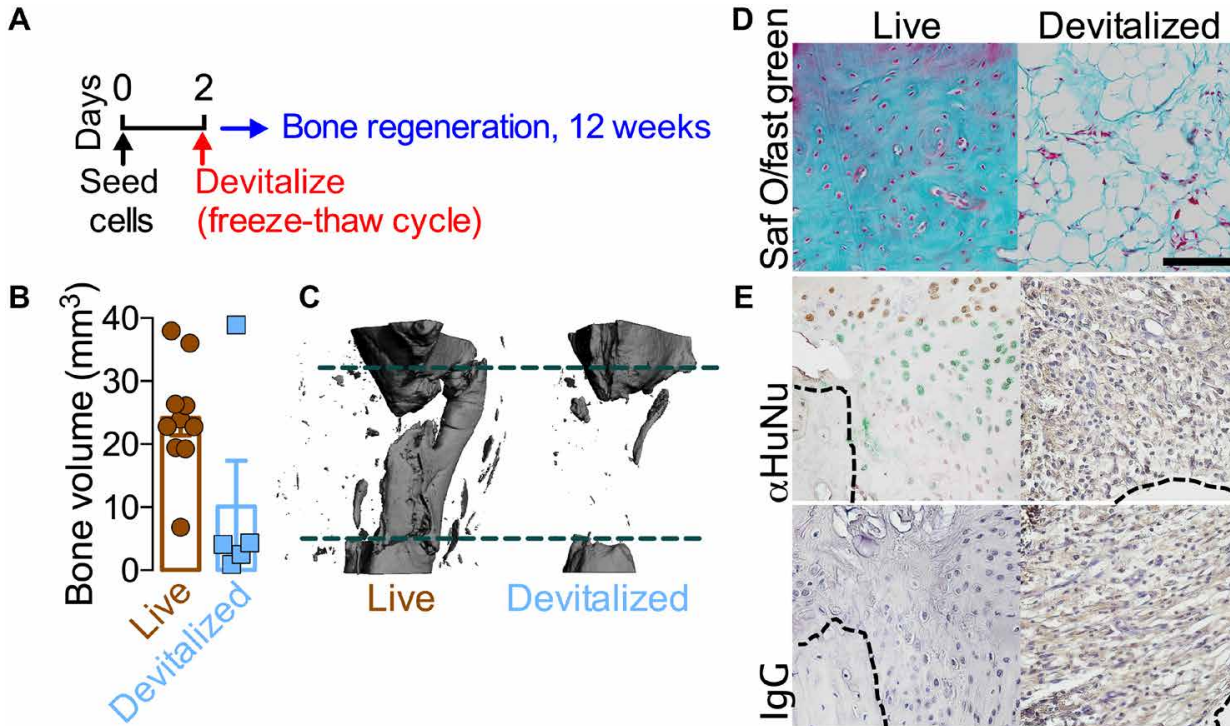

$\mathbf{F}$
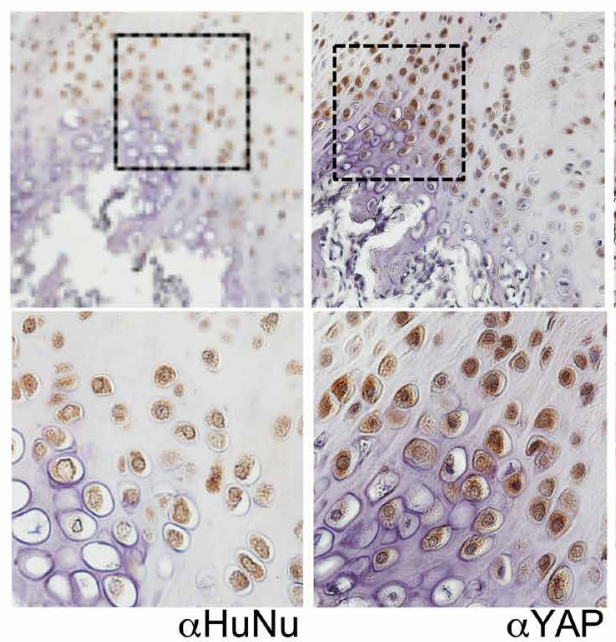

$\alpha$ YAP
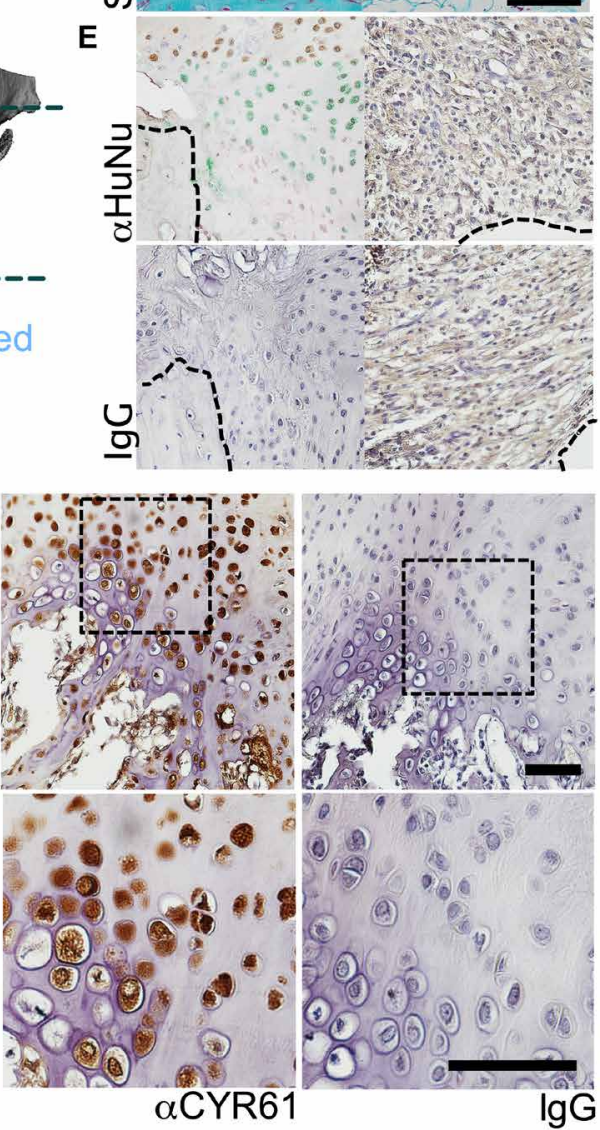

Fig. 3. Transplanted cell function. (A) Timeline depicting devitalization of mesenchymal condensations performed at day 2, before transplantation. (B and C) MicroCT analysis of bone formed at week 12 in live and devitalized groups ( $n=10$ and 5 for live and devitalized, respectively). Dashed lines illustrate the location of the native bone ends. (D) Representative safranin O/fast green staining at the center of the defects at week 12 ( $n=1$ to 3 per group). Representative samples selected on the basis of mean bone volume. Scale bar, $100 \mu \mathrm{m}$. (E) HuNu staining of live and devitalized samples (stiff plates) at week 12. DAB peroxidase produced a brown reaction product at locations of immunolabeled antigens. Dashed lines indicate the edges of the native cortical bone at the distal end of the defect. Devitalized samples exhibited some matrix-associated nonspecific staining, as shown in IgG controls. (F) Immunostaining of HuNU, YAP, and CYR61 in defects of the delayed loading group (live cells). In each case, DAB peroxidase was used to produce a brown reaction product at locations of immunolabeled antigens. Isotype-matched IgG controls were used to demonstrate specificity. Bottom row shows magnification of boxed areas. Scale bars, $100 \mu \mathrm{m}$.

and $1.8 \mu \mathrm{g}$ of TGF- $\beta 1$ per construct) for implantation after devitalization by freeze-thaw cycling (Fig. 3A). Using stiff plates, devitalized condensations elicited substantially reduced bone formation compared to live cell controls (Fig. 3, B and C, and movie S1), with fibrotic and adipocytic tissue filling the defect (Fig. 3D). Comparisons between live and devitalized samples were not assessed statistically due to surgical operation at a separate time but suggest a functional role of the transplanted cells.

To further elucidate transplanted cell fate and function, live human cells were immunolocalized by human nuclear antigen $(\mathrm{HuNu})$

staining (Fig. 3, E and F) (21). Viable human cells, morphologically identifiable as mature and hypertrophic chondrocytes, were actively engaged in endochondral ossification as late as week 12 in vivo (Fig. 3, E and F). In contrast, devitalized samples exhibited some nonspecific background staining, illustrated by immunoglobulin $\mathrm{G}$ ( IgG) controls, but no live cells were detected. In live samples, human hypertrophic chondrocytes also exhibited nuclearlocalized YAP protein and expression of the downstream angiogenic matricellular growth factor, CYR61 (Fig. 3F). We did not observe $\mathrm{HuNu}^{+}$osteoblasts, which stained $\mathrm{CYR}^{+} 1^{+}$but were $\mathrm{HuNu}^{-}$ (Fig. 3F, top).

\section{Endochondral matrix formation}

To determine the extent to which loading regulated endochondral lineage progression and matrix organization $(28,31)$, we performed histological staining at weeks 3, 4, 7, and 12 (Fig. 4 and figs. S5 to S8). The endochondral regenerate contained distinct bands of safranin $\mathrm{O}-$ positive cartilage featuring mature and hypertrophic chondrocytes, producing woven bone. Extensive sGAG staining was observed at early time points ( 3 and 4 weeks) and calcified cartilage and bone at weeks 7 and 12 (Fig. 4 and figs. S5 to S8). Both early and delayed loading enhanced and prolonged the chondral phase of endochondral ossification, as indicated by safranin $\mathrm{O}$ staining intensity (Fig. 4, A to C, and figs. S5 to S8). Local collagen organization was evaluated by polarized light analysis of picrosirius red-stained sections (28) at weeks 4 and 12 . There were no qualitative differences in collagen birefringence at week 4 (fig. S7), but both early and delayed loading qualitatively decreased collagen organization compared to the stiff controls at week 12 (Fig. 4D). This suggests that loading increased the proportion of immature woven bone through either increased de novo woven bone deposition or altered remodeling $(18,28)$.

\section{Recovery of mechanical properties}

As the principal test of any engineered tissue needs to be its functionality (5), we evaluated the degree of restoration of bone mechanical properties by torsion testing to failure at week 12 , in comparison with age-matched intact femora (Fig. 5). Despite enhanced bone formation, early loading failed to restore mechanical properties, whereas delayed loading significantly increased torsional stiffness 
Fig. 4. Endochondral matrix formation. Tile scan images of Safranin O/fast green-stained histological sections of representative samples from stiff, early, and delayed loading groups at (A) week 4 and (B) week 12. Scale bar, $3 \mathrm{~mm}$. All samples oriented distal (left) to proximal (right). Dotted lines in top left indicate the native cortical bone ends. Label " $\mathrm{dh}$ " indicates location of fixation plate drill holes. Scale bar, $3 \mathrm{~mm}$. (C) Magnified images of dashed boxed regions in (B) showing endochondral cartilage remnants at week 12 . Scale bar, $100 \mu \mathrm{m}$. Bottom row: Magnification of boxed regions in the top row. (D) Polarized light microscopy of picrosirius red-stained histological sections at week 12 . Increased birefringent intensity indicates increased collagen fibril organization associated with bone matrix remodeling to lamellar bone; reduced birefringence indicates greater amounts of woven bone. Scale bars, $100 \mu \mathrm{m}$. Bottom row: Magnification of boxed regions in upper row. All images were taken from a representative sample that most closely matched the average in vivo microCT morphometry of that group.

and maximum torque at failure compared to stiff plate controls $(P<0.05$; Fig. 5, A and B) and restored the torsional stiffness to that of intact limbs (dotted lines/gray shading indicate mean \pm SD). The ability of materials to resist deformation and failure is determined by intrinsic material properties and the material amount and distribution, which, for torsional loading, can be measured as the polar moment of inertia (pMOI). Using microCT, we quantified the minimum and average pMOI for each limb; each was significantly elevated by delayed loading $(P<0.05$; Fig. 5, C and D). Mechanical behavior was variable, particularly in the early group. To explain this, we performed a best subsets regression analysis to optimize the combination of factors that best correlate with mechanical behavior. The parameter set included average pMOI, minimum pMOI, bone volume, binary bridging score (indicated in Fig. 5 by shaded versus open data points; for data, see fig. S3, D and E), and average mineral density. The optimal model was determined by minimization of the Akaike's information criterion (AIC) (37). We then performed type II multivariate regression analyses to determine the amount of variability in the measured stiffness and maximum torque that is explained by the selected predictors $\left(R^{2}\right)$. For both maximum torque and torsional stiffness, minimum pMOI and bridging score were the best combined predictors from among the top five models (Fig. 5, E and F, and fig. S9). Together, these data indicate that mechanical loading regulated the amount and functionality of regenerated bone, formed

C

D
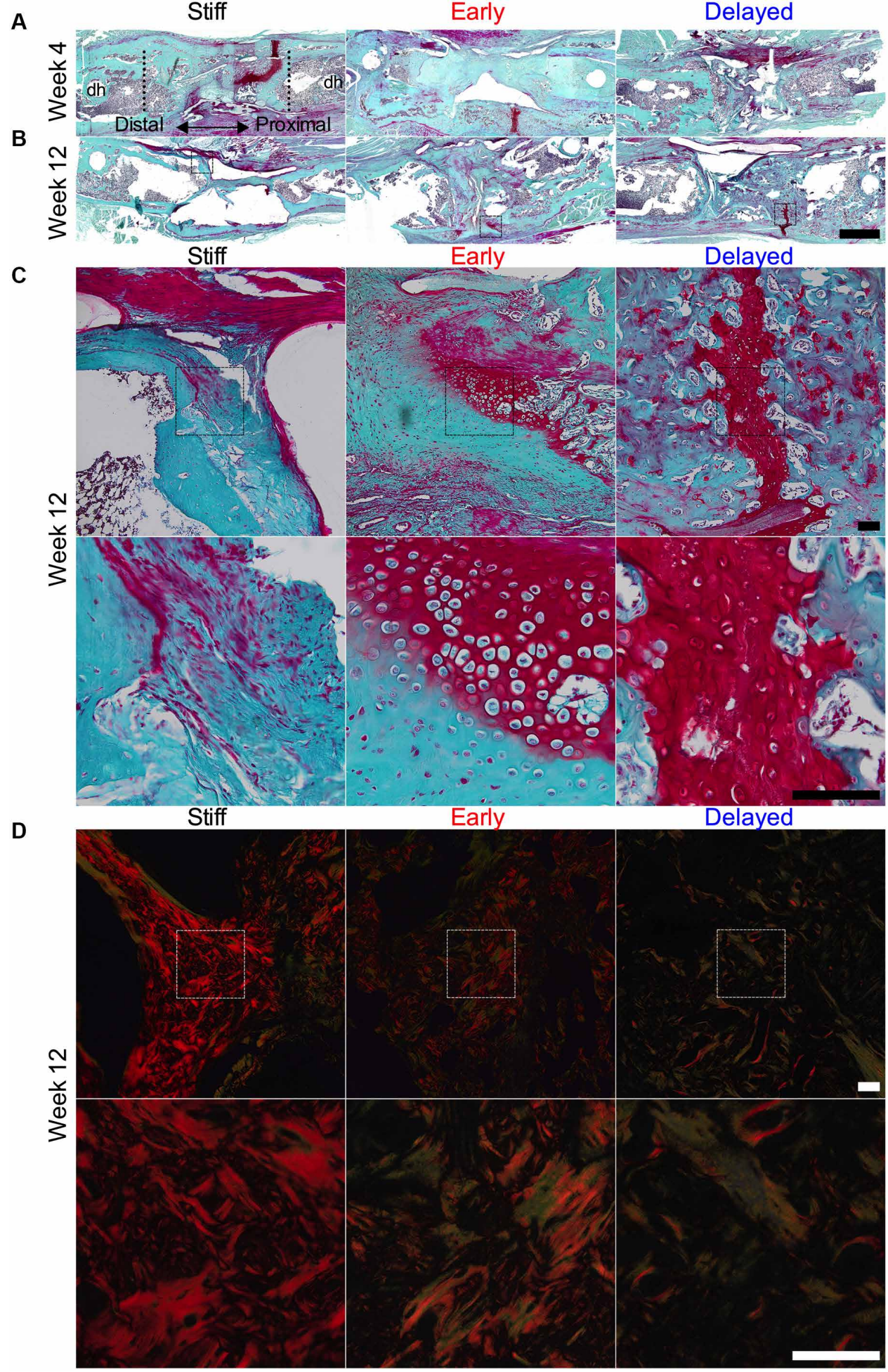

through endochondral ossification of transplanted mesenchymal condensations, but the mechanisms are unclear.

\section{Dual contrast-enhanced microCT imaging of vasculature and cartilage}

Angiogenesis is known to enhance and initiate cartilage callus ossification $(38-40)$. Therefore, we next hypothesized that mechanical 
A

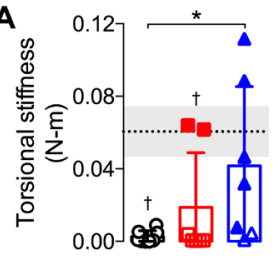

○ Stiff $\square$ Early

C

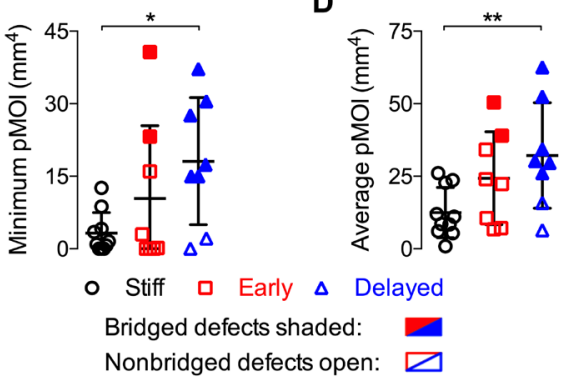

E

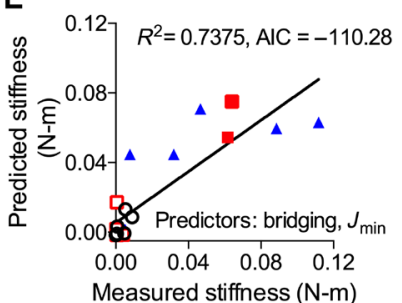

$\mathbf{F}$

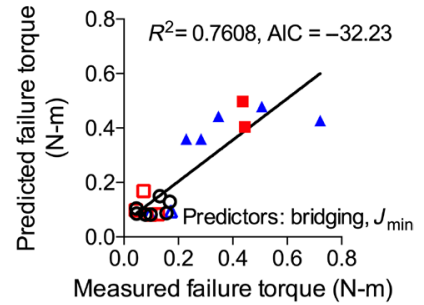

Fig. 5. Restoration of mechanical function. Structural mechanical properties were measured by torsion to failure at week 12 . Age-matched intact bone properties are shown as dotted lines/gray shading indicating mean \pm SD. Samples with full defect bridging are shown in filled data points; open data points indicate nonbridged samples. (A) Analysis of torsional stiffness, (B) maximum torque at failure, (C) minimum pMOI, and (D) average pMOI ( $n=8,7$, and 7 for stiff, early, and delayed, respectively). Best subsets regression analysis with lowest AIC value for measured and predicted torsional stiffness (E) and maximum torque at failure (F), indicating significant contributions of minimum pMOI $\left(J_{\mathrm{min}}\right)$ and binary bridging score. Error bars show means \pm SD with individual data points. Statistical comparisons between groups for each measure were performed by one-way ANOVA with Tukey's post hoc analyses, ${ }^{*} P<0.05 ;+P<0.05$ versus intact bone.

cues regulate endochondral bone formation, in part, by modulating neovascularization and subsequent cartilage template remodeling. To test this, we performed a second set of in vivo studies to quantify vascular invasion of the cartilage anlage by microCT angiography combined with contrast-enhanced cartilage imaging 3 weeks after the onset of loading for both early $(n=10)$ and delayed $(n=8)$ conditions. In this study, each animal was randomly assigned a loaded limb and a contralateral stiff plate control. Thus, animals in the early loading group were evaluated at week 3 and the delayed group at week 7 (Fig. 6, A and I). These time points were selected to capture the transient vascular network response to the dynamic mechanical environment $(18,40)$.

MicroCT angiography was performed by perfusing the vasculature with a lead chromate-based contrast agent (Microfil MV-122) to attenuate $\mathrm{x}$-rays in the vasculature (41-43) for three-dimensional (3D) quantification of blood vessel networks in and around the defect (Fig. 6 and fig. S10). Sequential microCT scanning of the perfused limbs before and after bone decalcification enabled independent
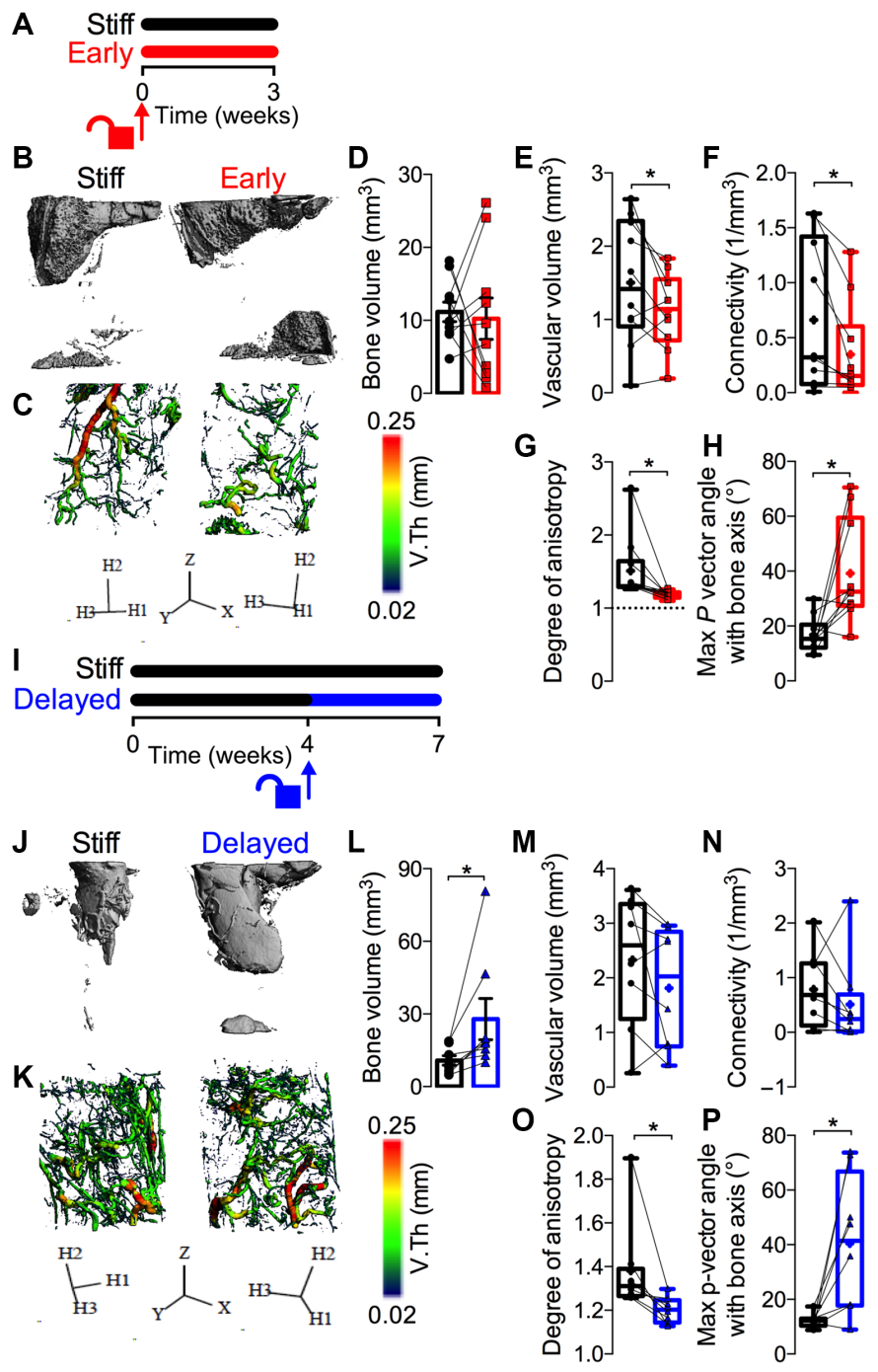

Fig. 6. Mechanical control of neovascularization. (A) Schematic of stiff versus early loading featuring compliant plate actuation at implantation and contrast agent perfused at week 3. (B and $\mathbf{C}$ ) Representative microCT reconstructions of bone (B) and blood vessels with local vessel diameter mapping (C) under stiff and early loading conditions at week 3. (D) Quantification of bone volume. (E to H) 3D vascular network morphometry quantifying vascular volume $(E)$, connectivity $(F)$, and vessel orientation and distribution, as measured by degree of anisotropy (G) and the angle with respect to the bone axis of the maximum principal eigenvector $(\mathrm{H} 2)$ of the mean intercept length $(\mathrm{MIL})$ tensor $(\mathrm{H})$, indicating the dominant direction of vessel orientation. Degree of anisotropy represents the ratio of the longest and shortest MIL eigenvalues; degree of anisotropy = 1 indicates isotropy. (I) Schematic of stiff versus delayed loading featuring compliant plate unlocking at week 4 and contrast agent perfused at week 7. ( $\mathbf{J}$ to $\mathbf{L}$ ) Representative microCT reconstructions of bone $(J)$ and blood vessels with local vessel diameter mapping $(K)$ under stiff and early loading conditions at week 7. (L) Quantification of bone volume. (M to $\mathbf{P})$ Vascular network morphometry measured by vascular volume (M), connectivity $(\mathrm{N})$, degree of anisotropy $(\mathrm{O})$, and maximum principal vector ( $\mathrm{p}$-vector) angle (P). Quantification is in a 5-mm ROI, paired data are either shown as means \pm SEM or superimposed on box plots displaying median as horizontal line, interquartile range as boxes, and minimum/maximum range as whiskers. Mean values are indicated by + . Comparisons between groups were evaluated by paired two-tailed Student's $t$ tests $\left({ }^{*} P<0.05\right)$. 
quantification of bone formation and vascularization in the same samples (18). In the defect ROI (5 mm Ø ROI), early loading did not alter bone formation at week 3 (Fig. 6, B and D), consistent with the independent previous results at week 4 (Fig. 2E). Early loading significantly inhibited vascular ingrowth $(P<0.05)$, blunting the predominantly axial orientation of the vessel network observed in the stiff group and producing a more isotropic distribution of orientations (Fig. 6, C and $\mathrm{E}$ to $\mathrm{H}$ ). In contrast, delayed loading enhanced bone formation at week 7 (Fig. 6, J and L), consistent with the independent previous results at week 8 (Fig. 2E). However, delayed loading did not alter vascular morphometry parameters other than reduced vessel anisotropy (Fig. 6, K and $\mathrm{M}$ to $\mathrm{P}$ ). Loading did not alter the vascular volume of the peripheral muscle $(7 \mathrm{~mm} \varnothing \mathrm{ROI})$, indicating a local effect of loading on vessel recruitment by the anlage, endothelial cell invasion, or neovessel integrity (fig. S10).

Next, to quantify $3 \mathrm{D}$ cartilage distribution, we equilibrated the vascular contrast agent-perfused samples with the cartilage contrast agent $\mathrm{CA}^{4+}$, which partitions at equilibrium with the negatively charged sGAGs and attenuates $\mathrm{x}$-rays proportional to sGAG concentration (44). Bone, vessel, and cartilage volumes were then assessed using various ROI analyses. We evaluated vascularization and cartilage formation within $1.5 \mathrm{~mm} \varnothing$ core and 5- to $1.5-\mathrm{mm}$ annular ROIs. Consistent with cartilage observed at 4 weeks, the core region had lower vascular volume in all groups (Fig. 7, A, B, D, and E), although the sGAG-positive tissue distribution was not significantly different between regions $(P=0.84$; Fig. $7, \mathrm{C}, \mathrm{F}, \mathrm{G}$, and $\mathrm{H})$. Differences in cartilage volume between the stiff plate and early loading groups in annular and core regions were not statistically significant for either loading regimen $(P=0.53$; Fig. $7, \mathrm{C}$ and $\mathrm{F})$.

\section{Temporal effects of load on progenitor lineage specification}

Together, these data suggest that the effects of mechanical loading regulate endochondral bone regeneration in a manner dependent on load timing and endochondral cell state at the time of load initiation. Therefore, to explore the temporal effects of mechanical loading on chondrogenic lineage commitment (gene expression and matrix production), we performed in vitro bioreactor experiments using hMSCs encapsulated in fibrin hydrogels exposed to $10 \%$ dynamic compression. The fibrin hydrogels were used to provide a matrix capable of in vitro loading, which was applied continuously for 5 weeks (early), for 2 weeks after a 3-week free swelling period (delayed), or for 2 weeks before a 3 -week free swelling period (reversed) and compared to 5 week free swelling controls (Fig. 8, A and B). post hoc comparisons)
Loading increased DNA content, indicating increased proliferation and/or maintenance of viability (Fig. 8C), but did not alter sGAG per DNA, and reduced total collagen per DNA content (Fig. 8C). Alcian blue staining revealed a large, rounded cell morphology and increased pericellular sGAG staining in response to loading (Fig. 8D), especially in early and reversed groups where loading was applied immediately after encapsulation, suggesting load-induced PCM deposition. To test this, we immunostained for PCM-exclusive collagen 6 and found that all loaded groups exhibited increased collagen 6 at the cell periphery, particularly in the groups loaded immediately, whereas free swelling controls were nearly devoid of collagen 6 (Fig. 8E). Collagen 6a1 (COL6a1) mRNA expression was significantly increased in early and delayed groups $(P<0.0001$; Fig. $8 \mathrm{~F})$, suggesting that mechanical load is needed to initiate and maintain COL6a1 expression, resulting in COL6a1 accumulation in groups loaded immediately. Collagen 6 is prevalent in the PCM of articular chondrocytes and mediates load-induced proliferation and chondrogenic gene expression (45). Consistent with this observation, mRNA expression of
A

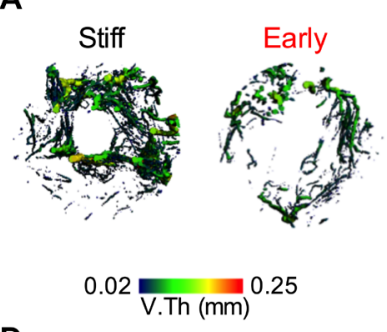

D

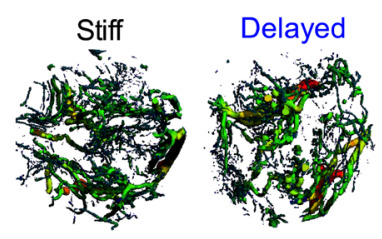

G Stiff

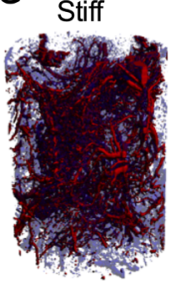

E

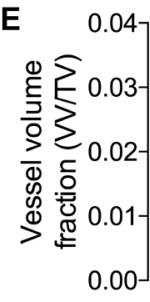

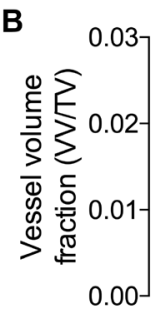
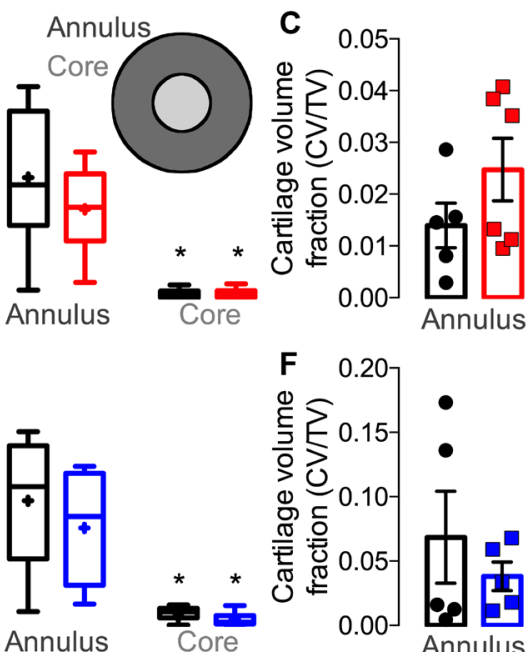
Annulus

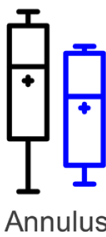

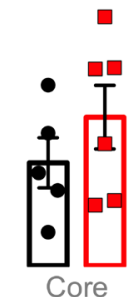

Core
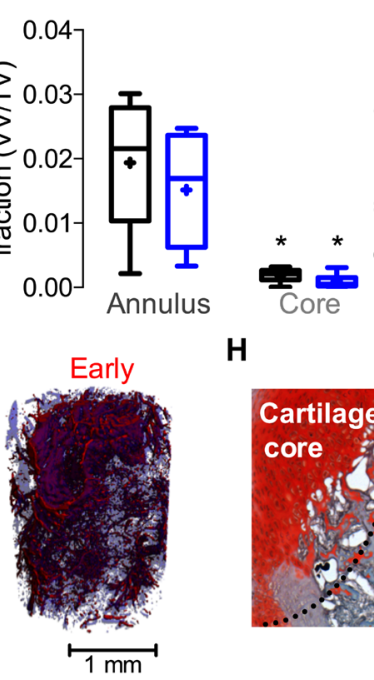

H
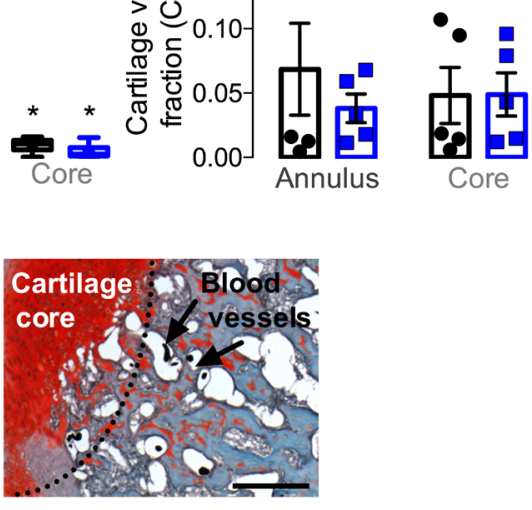

Fig. 7. Spatial distribution of neovascular and cartilaginous tissues. (A) Axial view of 3D neovessel diameter mapping under stiff and early loading conditions at week $3, n=10$. (B) ROI analysis to quantify vascular volume fraction in a 1.5-mm-diameter core region compared to a 5 - to $1.5-\mathrm{mm}$ annular region (inset). (C) Cationic (CA ${ }^{4+}$ ) $\mathrm{cartilage}^{-}$ contrast agent-enhanced microCT quantification of cartilage in annulus and core regions. $n=5$ to 6 . (D) Axial view of 3D neovessel diameter mapping under stiff and delayed loading conditions at week $7, n=8$. (E) ROI analysis of vascular volume fraction. (F) Cartilage contrast agent-enhanced microCT quantification of cartilage in annulus and core regions at week $7, n=5$ to 6 . (G) Representative image of coregistered contrast agent-enhanced cartilage with microCT angiography of neovasculature. Cartilage is shaded blue and vessels are red. (H) Safranin O/fast green-stained histological sections of vascular contrast agent-perfused tissues showing the avascular cartilage core and blood vessels in the surrounding tissue ( 3 weeks). Residual contrast agent exhibits thermal contraction during paraffin processing, visible as dark dots in vessel lumens. Scale bar, $100 \mu \mathrm{m}$. Data shown as means \pm SEM either with individual data points or with box plots displaying median as horizontal line, interquartile range as boxes, and minimum/ maximum range as whiskers. Mean values on box plots are indicated by + . $\left({ }^{*} P<0.05\right.$, two-way ANOVA with Tukey's 


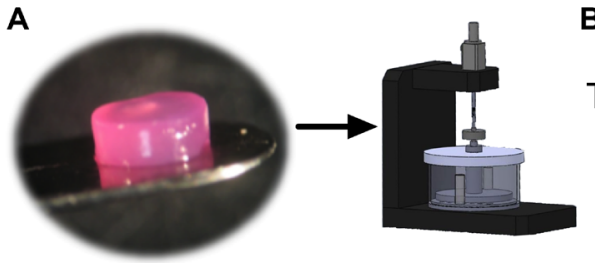

B
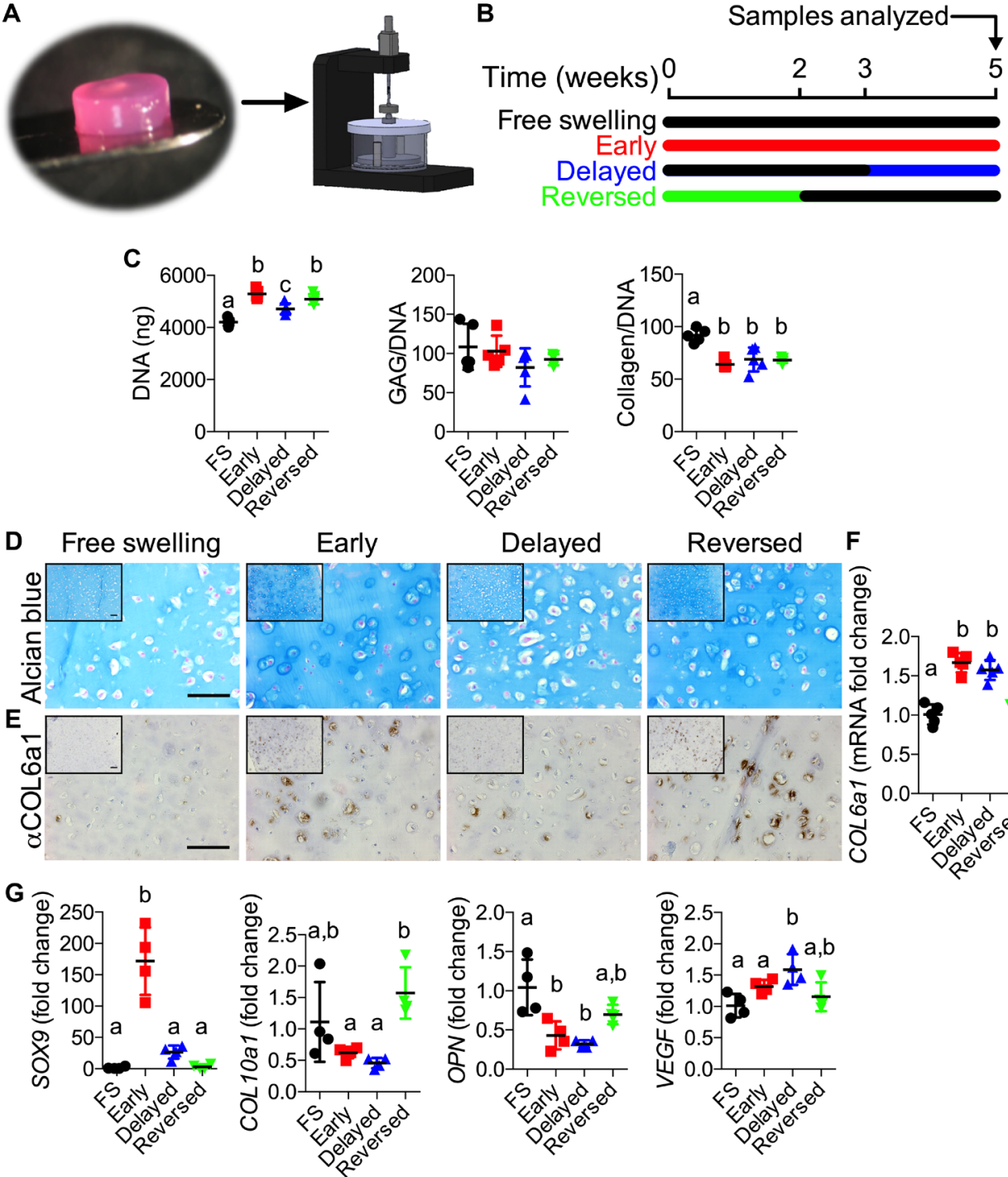

Fig. 8. In vitro analysis of mechanical load on chondrogenic lineage progression. (A) Photograph of hMSC-laden hydrogel and schematic of custom-made bioreactor applying dynamic compression. (B) Timeline of the four loading groups evaluated: free swelling (FS) controls, early loading (continuous for 5 weeks), delayed loading (free-swelling for 3 weeks followed by 2 weeks of loading), and reversed loading (loading for 2 weeks followed by 3 weeks of free swelling). (C) Quantification of DNA, sGAG, and total collagen content $(n=5)$. (D) Alcian blue and (E) pericellular COL6a1 immunostaining. DAB peroxidase was used to produce a brown reaction product at locations of immunolabeled antigen. Images shown at $20 \times$ with $10 \times$ insets. Scale bars, $100 \mu \mathrm{m}$. (F and G) qPCR at week 5 ( $n=4$ to 5 per group) of (F) COL6a1 and (G) SOX9, COL10a1, OPN, and VEGF. Relative expression was calculated as fold change over free swelling controls. Data are shown as means \pm SD with individual data points. Statistical comparisons between groups for each measure were performed by one-way ANOVA with Tukey's post hoc analyses, where groups sharing a letter $(a, b$, and $c)$ are not statistically different.

the master chondrogenic transcription factor $S O X 9$ increased in all loaded groups but was only significant when applied continuously (early loading, $P<0.0001$; Fig. 8G). This indicates a maintenance of chondrogenic gene expression that may explain the cartilage persistence seen in vivo in response to early loading. Similar trends were observed for SOX9 target genes aggrecan and COL2a1 (fig. S11, A and B). Consistent with the down-regulation of YAP and downstream target CYR61 observed with TGF- $\beta 1$-mediated chondrogenesis (Fig. 1), these genes were also significantly decreased with chondrogenic lineage commitment induced by loading $(P<0.05$; fig. S11B). Loading decreased

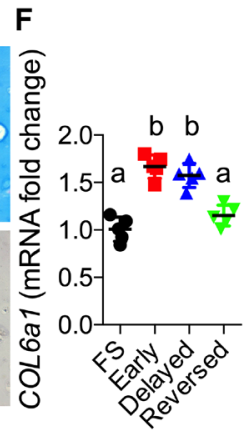

COL10a1 and osteopontin expression in groups where load was being applied at harvest (early and delayed), but not in the reversed group, suggesting that loading delayed chondrocyte hypertrophy (Fig. 8G). Vascular endothelial growth factor (VEGF) is an important factor for vascular invasion during endochondral healing and is necessary for remodeling of the fracture callus (46). Delayed loading significantly increased $V E G F$ expression $(P<0.01$; Fig. $8 \mathrm{G})$, consistent with our finding that delayed loading supported neovascularization and enhanced endochondral bone growth.

\section{DISCUSSION}

In development, mechanical forces caused by fetal motion in the womb are critical for proper bone and joint morphogenesis $(6,47)$. In adults, mechanical stability at a fracture site determines the mode of repair (intramembranous versus endochondral). We therefore reasoned that mechanical cues would also be important for recapitulating endochondral bone development for regeneration of large bone defects. Here, we evaluated the capacity of engineered mesenchymal condensations with local morphogen presentation to induce endochondral bone regeneration under varying conditions of in vivo mechanical loading. We found that mechanical loading enhanced endochondral bone formation in vivo and was essential to restore function to limbs with critical-sized bone defects. Mechanistically, loading regulated chondrogenesis and PCM formation in vitro and controlled cartilage persistence and neovascularization in vivo, dependent on load timing. Together, these data demonstrate the importance of mechanical cues for biomimetic bone tissue engineering and may have implications for other tissues whose form and function are dictated by mechanical stimuli during development and homeostasis.

Previous reports by our group $(18,28)$ and others $(48,49)$ investigated the effects of in vivo mechanical loading on recombinant BMP-2mediated bone defect repair. In these BMP studies, loading maximally induced an 18 to $20 \%$ increase in bone formation compared to stiff plate controls. By contrast, here, we used an endochondral regeneration approach to show that delayed mechanical loading increased bone volume by $181 \%$ compared to stiff controls, representing an order-ofmagnitude greater mechanoresponse than that under BMP-2 treatment. Further, the response to loading was greatest between weeks 4 and 8 , especially when loading was initiated at week 4 . Histologically, 
this corresponded with the timing of transition from hypertrophic cartilage to bone, suggesting that this stage of endochondral ossification is particularly responsive to mechanical forces. Functionally, mechanical loading was required to restore bone biomechanical properties, but mechanical outcomes exhibited high variability. Multivariate regression analysis (50) revealed that this variability was determined both by whether the defects were fully bridged with bone and the distribution of that bone in the defect. It further explains differences in mechanical outcomes between the early and delayed loading groups; although both loading conditions enhanced bone volume, the early loading failed to induce functional repair as a result of a low bridging rate due to pseudarthrotic cartilage. Together, these data demonstrate that mechanical cues are critical for restoration of bone form and function by endochondral ossification of engineered mesenchymal condensations.

Although many MSC transplantation studies observe rapid cell death (21), functional donor cell incorporation has been observed in studies investigating endochondral ossification (32-35). Here, the lack of bone formation after cell devitalization and immunolocalization of human antigens suggested that the transplanted human cells both participated in endochondral lineage progression and exhibited mechanosensitive gene activity upon hypertrophy. Recent studies demonstrate that hypertrophic chondrocytes can also transdifferentiate into osteoblasts during both developmental and reparative endochondral ossification $(36,51-54)$. Although we observed $\mathrm{HuNu}^{+}$ proliferative and hypertrophic chondrocytes, we did not detect $\mathrm{HuNu}^{+}$osteoblasts or evidence of hypertrophic chondrocyte-toosteoblast transformation.

Vascularization and anlage maturation are linked at both cellular and molecular scales and influence one another during endochondral ossification (38-40). For example, inhibition of angiogenesis can promote phenotypic stability of MSC-derived chondrocytes in vivo (55), whereas chondrocyte hypertrophy is, in part, responsible for neovessel recruitment (1). Further, we found previously that angiogenesis is influenced by mechanical conditions during bone defect repair (18) and can be modulated by scaffold architecture (56). Here, we found that mechanical loading did not affect vascular supply in the peripheral musculature, but both early and delayed loading increased the isotropy of the new vessel networks, particularly by decreasing the degree of axial orientation of the invading vasculature. Early loading reduced vascular volume and connectivity and, in some samples, caused pseudarthrotic nonunion, whereas delayed loading did not alter vascular volume or connectivity and enhanced defect bridging. Together, we speculate that loading may differentially regulate invasion by two distinct sources of angiogenic vessels: one from within the cortex and one from the surrounding musculature. Thus, loading may disproportionally impair or delay vascular invasion from the endocortical space while allowing transverse invasion from the surrounding musculature. These effects of loading on angiogenesis and cartilage transformation could be mediated by either mechanobiological or physical inhibition of neovessel network formation resulting in cartilage maintenance, or conversely, loading may delay chondrocyte hypertrophy and subsequent neovessel recruitment.

Further research will be required to dissect the distinct and/or interacting effects of loading on endochondral lineage progression versus angiogenesis; one approach is to study the tissues in isolation. Our bioreactor data, devoid of endothelium or blood supply, show that mechanical loading was needed to initiate and maintain COL6a1 expression and regulated both chondrogenesis and angiogenic growth factor expression. Collagen 6 is prevalent in the PCM of articular chondrocytes and functions to resist cellular deformation during cartilage matrix compression (57), mediating load-induced proliferation and chondrogenic gene expression (45). Because collagen 6 protein has a slower degradation rate than its mRNA, the immunostain represents the accumulation of matrix-embedded collagen 6 over the tissue history. Thus, early loading and reversed loading induced collagen 6 expression and deposition in the PCM, whereas neither stiff nor delayed loading induced COL6a1 deposition by week 5 . Supporting a transient induction of COL6a1 by loading, only the groups that had been loaded immediately before the time of assay at week 5 (early and delayed loading groups) showed up-regulation of COL6a1 message.

The molecular mechanisms that control endochondral ossification remain incompletely understood. Recent evidence from our laboratory and others implicates the transcriptional coactivator YAP as a mechanosensitive $(58,59)$, TGF- $\beta 1$-responsive $(60)$ regulator of progenitor cell lineage specification, promoting endochondral bone development (50) but inhibiting chondrogenesis (61). Consistent with these reports, we observed that YAP was consistently down-regulated with chondrogenic lineage commitment across multiple experiments, corroborating recent evidence that YAP inactivation is necessary to maintain the chondrogenic phenotype $(62,63)$. Our data further indicate that mechanical loading promotes chondrogenesis independent of YAP induction or transcriptional activation; however, potential roles of YAP as a mechanosensor in cartilage remain poorly understood. We conclude that early loading, before substantial matrix deposition, may have caused large cellular deformation, inducing production of a protective PCM and promoting and sustaining chondrogenic differentiation, leading to pseudarthrosis formation and inhibition of functional regeneration, whereas delayed loading supported angiogenesis and induced endochondral bone regeneration.

\section{Limitations}

The goals of this study were to mimic the cellular organization and local morphogen environment of the early developing limb for endochondral regeneration of large bone defects and to investigate the effects of in vivo mechanical cues on cell function and regenerative outcome. We found that mechanical cues are key mediators of endochondral regeneration, but the study had several limitations. First, although the use of hMSCs as a cell source provides translational potential, these cells are developmentally and functionally distinct from the mesenchymal cells that comprise the early limb bud (64). Further work will be necessary to test the functional consequences of these distinctions and/or identify other cell types better capable of reproducing the embryonic niche. Second, MSCs are known to exhibit donor-donor variability; we have directly investigated these effects in previous studies (16), but this study was not designed to evaluate differences between donors, and all experiments were performed using a single donor. We used athymic (RNU) rats to facilitate xenogeneic cell transplantation and assessment of functional hMSC engraftment (21), but this model may miss some immunomodulatory functions of the transplanted cells (65). However, potential translational application of these findings to the clinic will require further testing in a larger animal model but would likely involve autologous cell transplantation or immune-matched allogeneic cells, which would not illicit a T cell response. Clinical implementation of this mechanical stimulus-induced, development-inspired approach will require further research, beyond the scope of this study; 
however, these data support continued work on the roles of mechanical cues in development-mimetic tissue regeneration and may affect clinical management of challenging bone augmentation cases in the future. Further work will be necessary to identify the precise cellular, biochemical, and mechanical cues necessary for efficient regeneration and clinical translation in humans.

\section{MATERIALS AND METHODS Study design}

The main experimental design featured an 8 - $\mathrm{mm}$ critically sized rat femoral defect, each defect receiving three high-density hMSC sheets (a total of $6 \times 10^{6}$ cells and $1.8 \mu \mathrm{g}$ of TGF- $\beta 1$ ) contained within an electrospun, perforated poly-( $\varepsilon$-caprolactone) (PCL) nanofiber mesh tube (19), under three loading conditions. Control limbs (stiff) were stabilized with fixation plates that limited load transfer. Early loading limbs were stabilized by axially compliant fixation plates that allowed load transfer immediately upon implantation (early). Delayed loading limbs were stabilized by the same compliant plates implanted initially in a locked configuration to prevent loading, until surgical unlocking after 4 weeks to enable load transfer (delayed). Sample sizes were determined by power analyses $(\alpha=0.05$ and $\beta=0.2$ ), computed using effect sizes and variability described in previous studies $(17,18)$. Samples were assigned to animals to balance pairing of stiff, early, and delayed loading groups between limbs.

In additional animals, hMSC sheets were devitalized by three freeze-thaw cycles, combined in a PCL mesh tube, and placed into segmental defects under stiff fixation. As a clinical comparison, another set of animals were treated with rhBMP-2 (5 $\mu \mathrm{g} ; \mathrm{R} \& \mathrm{D}$ Systems) delivered in an absorbable type I collagen sponge (DSM) under stiff fixation (3). These were performed at a separate time from the main study and were therefore not included in quantitative statistical comparisons.

\section{hMSC isolation and expansion}

hMSCs were derived from the posterior iliac crest of a healthy male donor (41 years of age) using a protocol approved by the University Hospitals of Cleveland Institutional Review Board. Cells were isolated using a Percoll gradient (Sigma-Aldrich) and cultured in lowglucose Dulbecco's modified Eagle's medium (DMEM; SigmaAldrich) containing 10\% fetal bovine serum (Sigma-Aldrich), $1 \%$ penicillin/streptomycin (P/S; Thermo Fisher Scientific), and fibroblast growth factor-2 (10 ng/ml; R\&D Systems, Minneapolis, $\mathrm{MN})(15,16,66)$. Cells were verified to be negative for mycoplasma contamination during expansion and before in vivo implantation.

\section{GM synthesis and TGF- $\beta 1$ loading}

GMs (66-68) were synthesized from $11.1 \%(w / v)$ gelatin type A (Sigma-Aldrich) using a water-in-oil single emulsion technique and cross-linked for 4 hours with 1\% (w/v) genipin (Wako USA) (69). Hydrated GMs were light blue in color and spherical in shape with an average diameter of $52.9 \pm 40.2 \mu \mathrm{m}$ and a cross-linking density of $25.5 \pm 7.0 \%$. Growth factor-loaded microspheres were prepared by soaking cross-linked, ultraviolet (UV)-sterilized GMs in solution of rhTGF- $\beta 1$ ( $80 \mu \mathrm{g} / \mathrm{ml}$; PeproTech) in phosphate-buffered saline (PBS) for 2 hours at $37^{\circ} \mathrm{C}$. Unloaded microspheres without growth factor were hydrated similarly using only PBS. Sheet thickness was quantified by Fiji (Image J) on transverse sections.

\section{Microsphere-incorporated hMSC sheet preparation}

Expanded hMSCs $\left(2 \times 10^{6}\right.$ cells per sheet; passage 4$)$ were mixed with TGF- $\beta 1$-loaded GM (400 ng/mg; $1.5 \mathrm{mg}$ per sheet) in chemically defined medium [high-glucose DMEM (Sigma-Aldrich), $1 \%$ ITS $^{+}$Premix (Corning), $1 \mathrm{mM}$ sodium pyruvate (HyClone), $100 \mu \mathrm{M}$ nonessential amino acids (Lonza), $100 \mathrm{nM}$ dexamethasone (MP Biomedicals), $0.13 \mathrm{mM}$ L-ascorbic acid-2-phosphate (Wako USA), and 1\% P/S (Thermo Fisher Scientific)]. Five hundred microliters of the suspension was seeded onto the prewetted membrane of Transwell inserts (3- $\mu \mathrm{m}$ pore size and $12 \mathrm{~mm}$ in diameter; Corning) and allowed to self-assemble for 2 days. After 24 hours, medium in the lower compartment was replaced. Control sheets containing unloaded GM were prepared and cultured in a similar fashion. After 48 hours, hMSC sheets were harvested for implantation.

\section{Nanofiber mesh production}

Nanofiber meshes were formed by dissolving 12\% (w/v) PCL (SigmaAldrich) in 90/10 (v/v) hexafluoro-2-propanol/dimethylformamide (Sigma-Aldrich). The solution was electrospun at a rate of $0.75 \mathrm{ml} /$ hour onto a static aluminum collector. Sheets $(9 \mathrm{~mm}$ by $20 \mathrm{~mm}$ ) were cut from the product, perforated with a 1-mm biopsy punch (VWR), and glued into tubes around a 4.5- $\mathrm{mm}$ mandrel with UV glue (Dymax). Meshes were sterilized by $100 \%$ ethanol evaporation over $\sim 15$ hours under UV light overnight and washed three times with sterile PBS before implantation.

\section{Surgical procedure}

Critical-sized (8-mm) bilateral segmental defects were created in the femora of 14-week-old male RNU rats (Charles River Laboratories) under isoflurane anesthesia (22). Limbs were stabilized by custom internal fixation plates that allow controlled transfer of ambulatory loads in vivo (17) and secured to the femur by four bicortical miniature screws (J.I. Morris Co.). Animals were given subcutaneous injections of $0.04 \mathrm{mg} / \mathrm{kg}$ of buprenorphine every 8 hours for the first 48 hours postoperative and $0.013 \mathrm{mg} / \mathrm{kg}$ every 8 hours for the following 24 hours. All procedures were reviewed and approved by the Institutional Animal Care and Use Committee at the University of Notre Dame (Protocol no. 14-05-1778).

\section{Fixation plate mechanical characterization}

Characterization of the axial, torsional, and flexural plate stiffness was performed by screwing the plate onto two stainless steel 3.9-mm-diameter rods through the tapped holes in the plate. Axial tests were conducted in both the fixed-fixed and fixed-free configurations on all three plate configurations with a control rate of $0.02 \mathrm{~mm} / \mathrm{s}$ to a displacement of $1.2 \mathrm{~mm}$ for the unlocked compliant and $0.005 \mathrm{~mm} / \mathrm{s}$ to a displacement of $0.2 \mathrm{~mm}$ for stiff and locked compliant. Torsional tests were conducted with a control rate of $0.1 \%$ s to a displacement of $5^{\circ}$. Four-point bending tests were conducted using 3.9-mm square rods in the convex, concave, and side orientations on the three plate configurations with a control rate of $0.05 \mathrm{~mm} / \mathrm{s}$ to a displacement of $2 \mathrm{~mm}$ for the unlocked compliant and $0.05 \mathrm{~mm} / \mathrm{s}$ to a displacement of $1.5 \mathrm{~mm}$ for stiff and locked compliant. The stiffness under each loading condition was calculated as the slope of the linear region of the load-displacement curves.

\section{In vivo $x$-ray and microCT}

In vivo $\mathrm{x}$-rays and microCT scans were performed at 4,8 , and 12 weeks to determine bridging and assess bone volume of the 
Table 1. Oligonucleotide primer sequences for qRT-PCR. Fwd, forward; Rev, reverse.

\begin{tabular}{|c|c|c|c|}
\hline Gene & & Sequence $\left(5^{\prime}-3^{\prime}\right)$ & Accession number \\
\hline \multirow[t]{2}{*}{ SOX9 } & Fwd & CACACAGCTCACTCGACCTTG & NM_000346.3 \\
\hline & $\operatorname{Rev}$ & TTCGGTTATITTAGGATCATCTCG & \\
\hline & $\operatorname{Rev}$ & CACGATGCCTITCACCACGAC & \\
\hline COL2A1 & Fwd & GGAAACTITGCTGCCCAGATG & NM_001844.4 \\
\hline & $\operatorname{Rev}$ & TGGGCAGCTGGGGGTTCAGT & \\
\hline \multirow[t]{2}{*}{ RUNX2 } & Fwd & ACAGAACCACAAGTGCGGTGCAA & NM_001015051.3 \\
\hline & $\operatorname{Rev}$ & TGGCTGGTAGTGACCTGCGGA & \\
\hline$A L P$ & Fwd & CCACGTCTTCACATTTGGTG & NM_000478.4 \\
\hline \multirow[t]{2}{*}{ GAPDH } & Fwd & GGGGCTGGCATTGCCCTCAA & NM_002046.5 \\
\hline & $\operatorname{Rev}$ & GGCTGGTGGTCCAGGGGTCT & \\
\hline \multirow[t]{2}{*}{ YAP } & Fwd & CAACTCCAACCAGCAGCAACA & NM_001130145 \\
\hline & $\operatorname{Rev}$ & GCAGCCTCTCCTTCTCCATCTG & \\
\hline \multirow[t]{2}{*}{$T A Z$} & Fwd & ACCCACCCACGATGACCCCA & NM_015472 \\
\hline & $\operatorname{Rev}$ & GCACCCTAACCCCAGGCCAC & \\
\hline \multirow[t]{2}{*}{ CTGF } & Fwd & AGGAGTGGGTGTGTGACGA & NM_001901 \\
\hline & $\operatorname{Rev}$ & CCAGGCAGTTGGCTCTAATC & \\
\hline CYR61 & Fwd & GAGTGGGTCTGTGACGAGGAT & NM_001554 \\
\hline \multirow[t]{2}{*}{$A C A N$} & Fwd & TGCGGGTCAACAGTGCCTATC & NM_001135 \\
\hline & $\operatorname{Rev}$ & CACGATGCCTITCACCACGAC & \\
\hline \multirow[t]{2}{*}{ COL2a1 } & Fwd & GAAGAGTGGAGACTACTGG & NM_033150 \\
\hline & $\operatorname{Rev}$ & CAGATGTGTITCTTCTCCTTG & \\
\hline \multirow[t]{2}{*}{ OPN (SPP1) } & Fwd & GACCAAGGAAAACTCACTAC & NM_001251829 \\
\hline & $\operatorname{Rev}$ & CTGTTTAACTGGTATGGCAC & \\
\hline \multirow[t]{2}{*}{$R U N \times 2$} & Fwd & AAGCTTGATGACTCTAAACC & NM_001015051 \\
\hline & $\operatorname{Rev}$ & TCTGTAATCTGACTCTGTCC & \\
\hline \multirow[t]{2}{*}{ COL10a1 } & Fwd & GCTAGTATCCTTGAACTTGG & NM_000493 \\
\hline & $\operatorname{Rev}$ & CCTITACTCTITATGGTGTAGG & \\
\hline \multirow[t]{2}{*}{ VEGFA } & Fwd & AATGTGAATGCAGACCAAAG & NM_001204384 \\
\hline & $\operatorname{Rev}$ & GACTTATACCGGGATTTCTTG & \\
\hline \multirow[t]{2}{*}{ COL6a1 } & Fwd & ACAGTGACGAGGTGGAGATCA & NM_001848 \\
\hline & $\operatorname{Rev}$ & GATAGCGCAGTCGGTGTAGG & \\
\hline
\end{tabular}


defect, respectively. In vivo CT scans were performed on an Albira $\mathrm{Si}$ imaging system (Bruker) at $45 \mathrm{kVp}, 0.4 \mathrm{~mA}$, with best resolution $(125-\mu \mathrm{m}$ voxel size). Forty-five slices were analyzed in the center of each defect with a global threshold of 400 to determine bone volume. For the group treated with the rhBMP-2/absorbable collagen scaffold, microCT analysis was performed using a Scanco $\mu \mathrm{CT} 80$ system (Scanco Medical) at $70 \mathrm{kVp}, 114 \mu \mathrm{A}$, at a resolution of $39 \mu \mathrm{m}$ per voxel. For this group, 144 slices (144 slices $\times$ $39 \mu \mathrm{m}$ per voxel $=5.616 \mathrm{~mm}$ ) were analyzed in the center of each defect with a global threshold of 270. X-rays were taken using an Xtreme scanner (Bruker) at $45 \mathrm{kVp}, 0.4 \mathrm{~mA}$, with $2 \mathrm{~s}$ exposure time. A binary bridging was score was assigned by two independent, blinded observers and determined as mineralized tissue fully traversing the defect.

\section{Biomechanical testing}

Femora excised at 12 weeks were biomechanically tested in torsion to failure. Limbs were cleaned of soft tissue, and the fixation plate was carefully removed. Bone ends were potted in Wood's metal (Alfa Aesar), mounted on a Bose ElectroForce biaxial load frame system (ELF 330, Bose EnduraTEC) and tested to failure at a rate of $3 \%$. For each sample, maximum torque at failure was recorded, and torsional stiffness was determined as the slope of a $5^{\circ}$ linear region in the torque-rotation curve. Samples were compared to seven agematched, unoperated femora.

\section{Fibrin gel preparation and dynamic compression}

Expanded hMSCs (passage 4) were resuspended in a final hydrogel solution of fibrinogen $(50 \mathrm{mg} / \mathrm{ml})$ and thrombin $(2.5 \mathrm{U} / \mathrm{ml})$. Gel was pipetted into $5 \mathrm{~mm}$ diameter by $2 \mathrm{~mm}$ thickness cylindrical agarose molds to create uniform constructs with a total cell volume of about 600,000 each. Culture was maintained in chondrogenic medium, where fresh medium was supplied every 3 days. Dynamic unconfined compressive loading was applied to the constructs using a custommade bioreactor. Load was applied 2 hours/day, 5 days a week at $1 \mathrm{~Hz}$, and $10 \%$ strain after a $0.01 \mathrm{~N}$ preload was applied. Load was applied continuously for 5 weeks (early), for 2 weeks after a 3-week free swelling period (delayed), or for 2 weeks before a 3 -week free swelling period (reversed) in comparison to 5-week free swelling controls.

\section{Quantitative reverse transcription polymerase chain reaction analysis}

hMSC sheet halves and fibrin hydrogels were homogenized in TRI Reagent (Sigma-Aldrich) for subsequent total RNA extraction and complementary DNA (cDNA) synthesis (PrimeScript 1st strand cDNA Synthesis Kit; Takara Bio Inc.). One hundred nanograms of cDNA was amplified in duplicates in each 40-cycle reaction using a Mastercycler (Eppendorf) with annealing temperature set at $60^{\circ} \mathrm{C}$, SYBR Premix Ex Taq II (Takara), and custom-designed qRT-PCR primers (Table 1; Life Technologies). Transcript expression was normalized to GAPDH, and gene expression was calculated as fold change using the comparative $\mathrm{C}_{\mathrm{T}}$ method (70).

\section{MicroCT angiography}

The segmental defect surgery was repeated in 14-week-old male RNU rats (Charles River Laboratories) under isoflurane anesthesia. In each rat, a loaded limb (early or delayed) was paired with a contralateral control limb, stabilized by a stiff fixation plate. Two animals were lost in the delayed group due to plate complications. Contrast- enhanced microCT angiography was performed at week 3 in the early loading group and week 7 in the delayed group $(41,71)$. After perfusion, limbs were excised and scanned via microCT (as described above) with both bone and contrast agent intact. Limbs were then decalcified with Cal-Ex II fixative/decalcifier (Thermo Fisher Scientific) for 2 weeks, scans were repeated, and subtraction was used to distinguish between bone and vessel parameters in a 5-mm-diameter defect ROI and 7-mm-diameter total ROI. Three representative samples from each group, chosen on the basis of average microCT-computed vessel volume, were processed for histology.

\section{Statistical analysis}

Individual sample sizes and details of statistical analyses are specified in each figure caption. Briefly, bridging rates were assessed by $\chi^{2}$ test for trend, and comparisons among groups were assessed with individual $\chi^{2}$ tests and Bonferroni correction for multiple comparisons. Comparisons between groups were assessed by one- or two-way analysis of variance (ANOVA) with Tukey's multiple comparisons tests, as appropriate. Where necessary and appropriate, data were log-transformed to ensure normality and homoscedasticity before ANOVA. Normality of dependent variables and residuals was verified by D'Agostino-Pearson omnibus and Brown-Forsythe tests, respectively. For mechanical property regressions, we used a best subsets algorithm to determine the best predictors of maximum torque and stiffness from a subset of morphologic parameters measured, which included minimum or average pMOI $\left(J_{\min }\right.$ or $\left.J_{\text {avg }}\right)$, bone volume, bridging (binary score), and mineral density based on AIC (37). The lowest AIC selects the best model while giving preference to models with less parameters. Last, the overall best model for each predicted mechanical property was compared to its measured value using type II general linear regression. The sample sizes for microCT, mechanical testing, and contrast-enhanced angiography analyses were determined with $\mathrm{G}^{*}$ Power software (72) based on a power analysis using population SDs and estimated effect sizes from our previous studies $(18,73)$. The power analysis assumed a two-tailed $\alpha$ of 0.05 , power of 0.8 , and effect sizes ranging from 0.1 to 0.3 . Primary data are reported in data file $S 1$.

\section{SUPPLEMENTARY MATERIALS}

stm.sciencemag.org/cgi/content/full/11/495/eaav7756/DC1

Fig. S1. H\&E staining of murine embryonic limb buds and hMSC sheets used for preparation of mesenchymal condensations.

Fig. S2. Fixation plate characterization.

Fig. S3. Bone accumulation and bridging rates.

Fig. S4. MicroCT ROI analysis demonstrating formation of cortical and trabecular bone compartments.

Fig. S5. Full histological analysis of endochondral bone formation at week 4.

Fig. S6. Full histological analysis of endochondral bone formation at week 12.

Fig. S7. Collagen organization.

Fig. S8. Histology of contrast agent-perfused sections.

Fig. S9. Best subsets analysis of mechanical testing data, models 2 to 5 .

Fig. S10. Vasculature in defect periphery.

Fig. S11. mRNA expression of hMSCs in dynamically compressed hydrogels.

Movie S1. Fixation plate actuation and bone regeneration.

Data file S1. Primary data.

References (74-76)

\section{REFERENCES AND NOTES}

1. L. C. Gerstenfeld, D. M. Cullinane, G. L. Barnes, D. T. Graves, T. A. Einhorn, Fracture healing as a post-natal developmental process: Molecular, spatial, and temporal aspects of its regulation. J. Cell. Biochem. 88, 873-884 (2003). 
2. M. K. Sen, T. Miclau, Autologous iliac crest bone graft: Should it still be the gold standard for treating nonunions? Injury 38, S75-S80 (2007).

3. J. D. Boerckel, Y. M. Kolambkar, K. M. Dupont, B. A. Uhrig, E. A. Phelps, H. Y. Stevens, A. J. García, R. E. Guldberg, Effects of protein dose and delivery system on BMP-mediated bone regeneration. Biomaterials 32, 5241-5251 (2011).

4. M. J. Bosse, E. J. MacKenzie, J. F. Kellam, A. R. Burgess, L. X. Webb, M. F. Swiontkowski, R. W. Sanders, A. L. Jones, M. P. McAndrew, B. M. Patterson, M. L. McCarthy, T. G. Travison, R. C. Castillo, An analysis of outcomes of reconstruction or amputation after Leg-threatening injuries. N. Engl. J. Med. 347, 1924-1931 (2002).

5. D. L. Butler, S. A. Goldstein, F. Guilak, Functional tissue engineering: The role of biomechanics. J. Biomech. Eng. 122, 570-575 (2000).

6. V. Hamburger, M. Waugh, The primary development of the skeleton in nerveless and poorly innervated limb transplants of chick embryos. Physiol. Zool. 13, 367-382 (1940).

7. A. Hosseini, D. A. Hogg, The effects of paralysis on skeletal development in the chick embryo. I. General effects. J. Anat. 177, 159-168 (1991).

8. V. Lefebvre, P. Bhattaram, Vertebrate skeletogenesis. Curr. Top. Dev. Biol. 90, 291-317 (2010).

9. J. Kenwright, J. B. Richardson, A. E. Goodship, M. Evans, D. J. Kelly, A. J. Spriggins, J. H. Newman, S. J. Burrough, J. D. Harris, D. I. Rowley, Effect of controlled axial micromovement on healing of tibial fractures. Lancet 2, 1185-1187 (1986).

10. L. E. Claes, C. a Heigele, C. Neidlinger-Wilke, D. Kaspar, W. Seidl, K. J. Margevicius, P. Augat, Effects of mechanical factors on the fracture healing process. Clin. Orthop. Relat. Res. 355 , S132-S147 (1998).

11. D. Lacroix, P. J. Prendergast, A mechano-regulation model for tissue differentiation during fracture healing: Analysis of gap size and loading. J. Biomech. 35, 1163-1171 (2002).

12. L. Claes, K. Eckert-Hübner, P. Augat, The effect of mechanical stability on local vascularization and tissue differentiation in callus healing. J. Orthop. Res. 20, 1099-1105 (2002).

13. D. E. Ingber, V. C. Mow, D. Butler, L. Niklason, J. Huard, J. Mao, I. Yannas, D. Kaplan, G. Vunjak-Novakovic, Tissue engineering and developmental biology: Going biomimetic. Tissue Eng. 12, 3265-3283 (2006).

14. E. Alsberg, K. W. Anderson, A. Albeiruti, J. A. Rowley, D. J. Mooney, Engineering growing tissues. Proc. Natl. Acad. Sci. U.S.A. 99, 12025-12030 (2002).

15. L. D. Solorio, E. L. Vieregge, C. D. Dhami, P. N. Dang, E. Alsberg, Engineered cartilage via self-assembled hMSC sheets with incorporated biodegradable gelatin microspheres releasing transforming growth factor- $\beta 1$. J. Control. Release 158, 224-232 (2012).

16. L. D. Solorio, C. D. Dhami, P. N. Dang, E. L. Vieregge, E. Alsberg, Spatiotemporal regulation of chondrogenic differentiation with controlled delivery of transforming growth factor- $\beta 1$ from gelatin microspheres in mesenchymal stem cell aggregates. Stem Cells Transl. Med. 1, 632-639 (2012).

17. J. D. Boerckel, K. M. Dupont, Y. M. Kolambkar, A. S. P. Lin, R. E. Guldberg, In vivo model for evaluating the effects of mechanical stimulation on tissue-engineered bone repair. J. Biomech. Eng. 131, 084502 (2009).

18. J. D. Boerckel, B. A. Uhrig, N. J. Willett, N. Huebsch, R. E. Guldberg, Mechanical regulation of vascular growth and tissue regeneration in vivo. Proc. Natl. Acad. Sci. U.S.A. 108, E674-E680 (2011).

19. Y. M. Kolambkar, K. M. Dupont, J. D. Boerckel, N. Huebsch, D. J. Mooney, D. W. Hutmacher, R. E. Guldberg, An alginate-based hybrid system for growth factor delivery in the functional repair of large bone defects. Biomaterials 32, 65-74 (2011).

20. Y. M. Kolambkar, J. D. Boerckel, K. M. Dupont, M. Bajin, N. Huebsch, D. J. Mooney, D. W. Hutmacher, R. E. Guldberg, Spatiotemporal delivery of bone morphogenetic protein enhances functional repair of segmental bone defects. Bone 49, 485-492 (2011).

21. K. M. Dupont, K. Sharma, H. Y. Stevens, J. D. Boerckel, A. J. García, R. E. Guldberg, Human stem cell delivery for treatment of large segmental bone defects. Proc. Natl. Acad. Sci. U.S.A. 107, 3305-3310 (2010).

22. M. E. Oest, K. M. Dupont, H.-Y. Kong, D. J. Mooney, R. E. Guldberg, Quantitative assessment of scaffold and growth factor-mediated repair of critically sized bone defects. J. Orthop. Res. 25, 941-950 (2007).

23. Y. Han, V. Lefebvre, L-Sox 5 and Sox 6 drive expression of the aggrecan gene in cartilage by securing binding of Sox9 to a far-upstream enhancer. Mol. Cell. Biol. 28, 4999-5013 (2008).

24. V. Lefebvre, W. Huang, V. R. Harley, P. N. Goodfellow, B. de Crombrugghe, SOX9 is a potent activator of the chondrocyte-specific enhancer of the pro alpha1(II) collagen gene. Mol. Cell. Biol. 17, 2336-2346 (1997).

25. C. I. Lorda-Diez, J. A. Montero, J. A. Garcia-Porrero, J. M. Hurle, Tgf $\beta 2$ and 3 are coexpressed with their extracellular regulator $L t b p 1$ in the early limb bud and modulate mesodermal outgrowth and BMP signaling in chicken embryos. BMC Dev. Biol. 10, 69 (2010).
26. A. E. Goodship, J. L. Cunningham, J. Kenwright, Strain rate and timing of stimulation in mechanical modulation of fracture healing. Clin. Orthop. Relat. Res. S105-S115 (1998).

27. A. M. McDermott, D. E. Mason, A. S. P. Lin, R. E. Guldberg, J. D. Boerckel, Influence of structural load-bearing scaffolds on mechanical load-and BMP-2-mediated bone regeneration. J. Mech. Behav. Biomed. Mater. 62, 169-181 (2016).

28. J. D. Boerckel, Y. M. Kolambkar, H. Y. Stevens, A. S. P. Lin, K. M. Dupont, R. E. Guldberg, Effects of in vivo mechanical loading on large bone defect regeneration. J. Orthop. Res. 30, 1067-1075 (2012).

29. T. Wehner, U. Wolfram, T. Henzler, F. Niemeyer, L. Claes, U. Simon, Internal forces and moments in the femur of the rat during gait. J. Biomech. 43, 2473-2479 (2010).

30. B. S. Klosterhoff, K. Ghee Ong, L. Krishnan, K. M. Hetzendorfer, Y.-H. Chang, M. G. Allen, R. E. Guldberg, N. J. Willett, Wireless implantable sensor for noninvasive, longitudinal quantification of axial strain across rodent long bone defects. J. Biomech. Eng. 139, 111004 (2017).

31. K. T. S. Palomares, R. E. Gleason, Z. D. Mason, D. M. Cullinane, T. A. Einhorn, L. C. Gerstenfeld, E. F. Morgan, Mechanical stimulation alters tissue differentiation and molecular expression during bone healing. J. Orthop. Res. 27, 1123-1132 (2009).

32. F. E. Freeman, A. B. Allen, H. Y. Stevens, R. E. Guldberg, L. M. McNamara, Effects of in vitro endochondral priming and pre-vascularisation of human MSC cellular aggregates in vivo. Stem Cell Res. Ther. 6, 218 (2015)

33. C. Scotti, B. Tonnarelli, A. Papadimitropoulos, A. Scherberich, S. Schaeren, A. Schauerte, J. Lopez-Rios, R. Zeller, A. Barbero, I. Martin, Recapitulation of endochondral bone formation using human adult mesenchymal stem cells as a paradigm for developmental engineering. Proc. Natl. Acad. Sci. U.S.A. 107, 7251-7256 (2010).

34. P. N. Dang, S. Herberg, D. Varghai, H. Riazi, D. Varghai, A. McMillan, A. Awadallah, L. M. Phillips, O. Jeon, M. K. Nguyen, Endochondral ossification in critical-sized bone defects via readily implantable scaffold-free stem cell constructs. Stem Cells Transl. Med. 6, 1644-1659 (2017).

35. N. Harada, Y. Watanabe, K. Sato, S. Abe, K. Yamanaka, Y. Sakai, T. Kaneko, T. Matsushita, Bone regeneration in a massive rat femur defect through endochondral ossification achieved with chondrogenically differentiated MSCs in a degradable scaffold. Biomaterials 35, 7800-7810 (2014).

36. C. S. Bahney, D. P. Hu, A. J. Taylor, F. Ferro, H. M. Britz, B. Hallgrimsson, B. Johnstone, T. Miclau, R. S. Marcucio, Stem cell-derived endochondral cartilage stimulates bone healing by tissue transformation. J. Bone Miner. Res. 29, 1269-1282 (2014).

37. H. Akaike, A new look at the statistical model identification. IEEE Trans. Automat. Contr. 19, 716-723 (1974).

38. S. K. Ramasamy, A. P. Kusumbe, L. Wang, R. H. Adams, Endothelial Notch activity promotes angiogenesis and osteogenesis in bone. Nature 507, 376-380 (2014).

39. H.-P. Gerber, T. H. Vu, A. M. Ryan, J. Kowalski, Z. Werb, N. Ferrara, VEGF couples hypertrophic cartilage remodeling, ossification and angiogenesis during endochondral bone formation. Nat. Med. 5, 623-628 (1999).

40. C. Huang, V. P. Ness, X. Yang, H. Chen, J. Luo, E. B. Brown, X. Zhang, Spatiotemporal analyses of osteogenesis and angiogenesis via intravital imaging in cranial bone defect repair. J. Bone Miner. Res. 30, 1217-1230 (2015).

41. J. D. Boerckel, D. E. Mason, A. M. McDermott, E. Alsberg, Microcomputed tomography: Approaches and applications in bioengineering. Stem Cell Res. Ther. 5, 144 (2014).

42. H. A. Cole, M. Yuasa, G. Hawley, J. M. M. Cates, J. S. Nyman, J. G. Schoenecker, Differential development of the distal and proximal femoral epiphysis and physis in mice. Bone $\mathbf{5 2}$, 337-346 (2013).

43. M. Yuasa, N. A. Mignemi, J. V. Barnett, J. M. M. Cates, J. S. Nyman, A. Okawa, T. Yoshii, H. S. Schwartz, C. M. Stutz, J. G. Schoenecker, The temporal and spatial development of vascularity in a healing displaced fracture. Bone 67, 208-221 (2014).

44. L. N. M. Hayward, C. M. J. de Bakker, L. C. Gerstenfeld, M. W. Grinstaff, E. F. Morgan, Assessment of contrast-enhanced computed tomography for imaging of cartilage during fracture healing. J. Orthop. Res. 31, 567-573 (2013).

45. P. Smeriglio, L. Dhulipala, J. H. Lai, S. B. Goodman, J. L. Dragoo, R. L. Smith, W. J. Maloney, F. Yang, N. Bhutani, Collagen VI enhances cartilage tissue generation by stimulating chondrocyte proliferation. Tissue Eng. Part A 21, 840-849 (2015).

46. K. D. Hankenson, M. Dishowitz, C. Gray, M. Schenker, Angiogenesis in bone regeneration. Injury 42, 556-561 (2011).

47. S. W. Verbruggen, J. H. W. Loo, T. T. A. Hayat, J. V. Hajnal, M. A. Rutherford, A. T. M. Phillips, N. C. Nowlan, Modeling the biomechanics of fetal movements. Biomech. Model. Mechanobiol. 15, 995-1004 (2016).

48. V. Glatt, M. Miller, A. Ivkovic, F. Liu, N. Parry, D. Griffin, M. Vrahas, C. Evans, Improved healing of large segmental defects in the rat femur by reverse dynamization in the presence of bone morphogenetic protein-2. J. Bone Joint Surg. Am. 94, 2063-2073 (2012).

49. V. Glatt, N. Bartnikowski, N. Quirk, M. Schuetz, C. Evans, Reverse dynamization: influence of fixator stiffness on the mode and efficiency of large-bone-defect healing at different doses of rhBMP-2. J. Bone Joint Surg. Am. 98, 677-687 (2016). 
50. C. D. Kegelman, D. E. Mason, J. H. Dawahare, D. J. Horan, G. D. Vigil, S. S. Howard, A. G. Robling, T. M. Bellido, J. D. Boerckel, Skeletal cell YAP and TAZ combinatorially promote bone development. FASEB J. 32, 2706-2721 (2018).

51. L. Yang, K. Y. Tsang, H. C. Tang, D. Chan, K. S. Cheah, Hypertrophic chondrocytes can become osteoblasts and osteocytes in endochondral bone formation. Proc. Natl. Acad. Sci. U.S.A. 111, 12097-12102 (2014).

52. X. Zhou, K. von der Mark, S. Henry, W. Norton, H. Adams, B. de Crombrugghe, Chondrocytes transdifferentiate into osteoblasts in endochondral bone during development, postnatal growth and fracture healing in mice. PLOS Genet. 10, e1004820 (2014).

53. C. Bahney, D. Hu, F. Ferro, A. Taylor, T. Miclau, R. Marcucio, Transdifferentiation of hypertrophic chondrocytes during endochondral bone repair by activation of pluripotent stem cell programs (216.1). FASEB J. 28, 216.1 (2014).

54. D. Giovannone, S. Paul, S. Schindler, C. Arata, D. T. Farmer, P. Patel, J. Smeeton, J. G. Crump, Programmed conversion of hypertrophic chondrocytes into osteoblasts and marrow adipocytes within zebrafish bones. eLife 8, e42736 (2019).

55. A. Marsano, C. M. M. da Cunha, S. Ghanaati, S. Gueven, M. Centola, R. Tsaryk, M. Barbeck, C. Stuedle, A. Barbero, U. Helmrich, Spontaneous in vivo chondrogenesis of bone marrow-derived mesenchymal progenitor cells by blocking vascular endothelial growth factor signaling. Stem Cells Transl. Med. 5, 1730-1738 (2016).

56. A. C. Daly, P. Pitacco, J. Nulty, G. M. Cunniffe, D. J. Kelly, 3D printed microchannel networks to direct vascularisation during endochondral bone repair. Biomaterials 162, 34-46 (2018).

57. L. G. Alexopoulos, I. Youn, P. Bonaldo, F. Guilak, Developmental and osteoarthritic changes in Col6a1 -knockout mice: Biomechanics of type VI collagen in the cartilage pericellular matrix. Arthritis Rheum. 60, 771-779 (2009).

58. S. Dupont, L. Morsut, M. Aragona, E. Enzo, S. Giulitti, M. Cordenonsi, F. Zanconato, J. Le Digabel, M. Forcato, S. Bicciato, N. Elvassore, S. Piccolo, Role of YAP/TAZ in mechanotransduction. Nature 474, 179-183 (2011).

59. D. E. Mason, J. M. Collins, J. H. Dawahare, T. D. Nguyen, Y. Lin, S. L. Voytik-Harbin, P. Zorlutuna, M. C. Yoder, J. D. Boerckel, YAP and TAZ limit cytoskeletal and focal adhesion maturation to enable persistent cell motility. J. Cell Biol. 1369-1389 (2019).

60. D.-E. Pefani, D. Pankova, A. G. Abraham, A. M. Grawenda, N. Vlahov, S. Scrace, TGF- $\beta$ targets the Hippo pathway scaffold RASSF1A to facilitate YAP/SMAD2 nuclear translocation. Mol. Cell 63, 156-166 (2016).

61. A. Karystinou, A. J. Roelofs, A. Neve, F. P. Cantatore, H. Wackerhage, C. De Bari, Yes-associated protein (YAP) is a negative regulator of chondrogenesis in mesenchymal stem cells. Arthritis Res. Ther. 17, 147 (2015).

62. Y. Deng, A. Wu, P. Li, G. Li, L. Qin, H. Song, K. Kingston, M. Correspondence, K. K. Mak, Yap1 regulates multiple steps of chondrocyte differentiation during skeletal development and bone repair. Cell Rep. 14, 2224-2237 (2016).

63. W. Zhong, Y. Li, L. Li, W. Zhang, S. Wang, X. Zheng, YAP-mediated regulation of the chondrogenic phenotype in response to matrix elasticity. J. Mol. Histol. 44, 587-595 (2013).

64. G. Nusspaumer, S. Jaiswal, A. Barbero, R. Reinhardt, D. Ishay Ronen, A. Haumer, T. Lufkin, I. Martin, R. Zeller, Ontogenic identification and analysis of mesenchymal stromal cell populations during mouse limb and long bone development. Stem Cell Reports $\mathbf{9}$, 1124-1138 (2017).

65. C. Gotherstrom, O. Ringden, M. Westgren, C. Tammik, K. Le Blanc, Immunomodulatory effects of human foetal liver-derived mesenchymal stem cells. Bone Marrow Transplant. 32, 265-272 (2003).

66. P. N. Dang, N. Dwivedi, L. M. Phillips, X. Yu, S. Herberg, C. Bowerman, L. D. Solorio, W. L. Murphy, E. Alsberg, Controlled dual growth factor delivery from microparticles incorporated within human bone marrow-derived mesenchymal stem cell aggregates for enhanced bone tissue engineering via endochondral ossification. Stem Cells Transl. Med. 5, 206-217 (2016).

67. L. D. Solorio, A. S. Fu, R. Hernández-Irizarry, E. Alsberg, Chondrogenic differentiation of human mesenchymal stem cell aggregates via controlled release of TGF- $\beta 1$ from incorporated polymer microspheres. J. Biomed. Mater. Res. A 92, 1139-1144 (2010).

68. P. N. Dang, L. D. Solorio, E. Alsberg, Driving cartilage formation in high-density human adipose-derived stem cell aggregate and sheet constructs without exogenous growth factor delivery. Tissue Eng. Part A 20, 3163-3175 (2014).

69. S. Herberg, D. Varghai, Y. Cheng, A. D. Dikina, P. N. Dang, M. W. Rolle, E. Alsberg, High-density human mesenchymal stem cell rings with spatiotemporally-controlled morphogen presentation as building blocks for engineering bone diaphyseal tissue. Nanotheranostics 2, 128-143 (2018).

70. T. D. Schmittgen, K. J. Livak, Analyzing real-time $P C R$ data by the comparative $C_{T}$ method. Nat. Protoc. 3, 1101-1108 (2008).

71. C. L. Duvall, W. R. Taylor, D. Weiss, R. E. Guldberg, Quantitative microcomputed tomography analysis of collateral vessel development after ischemic injury. Am. J. Physiol. Heart Circ. Physiol. 287, H302-H310 (2004).

72. F. Faul, E. Erdfelder, A. Buchner, A.-G. Lang, Statistical power analyses using G* Power 3.1: Tests for correlation and regression analyses. Behav. Res. Methods 41, 1149-1160 (2009).

73. B. A. Uhrig, J. D. Boerckel, N. J. Willett, M.-T. A. Li, N. Huebsch, R. E. Guldberg, Recovery from hind limb ischemia enhances rhBMP-2-mediated segmental bone defect repair in a rat composite injury model. Bone 55, 420-417 (2013).

74. Y.-J. Kim, R. L. Y. Sah, J.-Y. Doong, A. J. Grodzinsky, Fluorometric assay of DNA in cartilage explants using Hoechst 33258. Anal. Biochem. 174, 168-176 (1988).

75. N. Y. Ignat'eva, N. A. Danilov, S. V. Averkiev, M. V. Obrezkova, V. V. Lunin, E. N. Sobol', Determination of hydroxyproline in tissues and the evaluation of the collagen content of the tissues. J. Anal. Chem. 62, 51-57 (2007).

76. R. C. Stewart, P. N. Bansal, V. Entezari, H. Lusic, R. M. Nazarian, B. D. Snyder, M. W. Grinstaff, Contrast-enhanced CT with a high-affinity cationic contrast agent for imaging ex vivo bovine, intact ex vivo rabbit, and in vivo rabbit cartilage. Radiology 266, 141-150 (2013).

Acknowledgments: We thank the staff of the Freimann Life Science Center (FLSC) at the University of Notre Dame for animal care and husbandry, the staff of the Notre Dame Integrated Imaging Facility (NDIIF) for imaging support, and A. Awadallah of the Case Western Reserve University Histology Core Facility for technical support. We thank G. Niebur, University of Notre Dame, for insightful comments on the manuscript. Funding: This work was supported by the Naughton Foundation (to A.M.M.), the Indiana Clinical and Translational Sciences Institute, grant number UL1TR001108 from the NIH (to J.D.B.), the American Heart Association, grant number 16SDG31230034 (to J.D.B.), the National Science Foundation grant number 1435467 (to J.D.B.), the NIH's National Institute of Arthritis and Musculoskeletal and Skin Diseases under award numbers R01AR066193, R01AR063194, and R01AR069564 (to E.A.), the NIH's National Institute of Biomedical Imaging \& Bioengineering under award number R01EB023907 (to E.A.), the NIH's National Institute of Dental and Craniofacial Research under award number 5F32DE024712 (to S.H.), the NIH's National Heart, Lung, and Blood Institute under award number T32HL134622 (to R.T.), and the Ohio Biomedical Research Commercialization Program under award number TECG20150782 (to E.A.). The contents of this publication are solely the responsibility of the authors and do not necessarily represent the official views of the $\mathrm{NIH}$, the National Science Foundation, or other funding agencies. Author contributions: E.A. and J.D.B. conceived and supervised the research. A.M.M., S.H., E.A., and J.D.B. designed the experiments, analyzed the data, and wrote the paper. All authors collected data and commented on and approved the final manuscript. Competing interests: E.A. and Case Western Reserve University have patent protection including U.S. patent number 10,030,228 "Scaffold-free tissue constructs" and others pending surrounding the cellular condensation methodology and technology (filing numbers 15/258,666; 15/698,429; 16/044,182; and PCT/US2019/026678. All other authors declare that they have no competing financial interests. Data and materials availability: All data associated with this study are present in the paper or the Supplementary Materials.

\section{Submitted 9 November 2018}

Accepted 13 May 2019

Published 5 June 2019

10.1126/scitransImed.aav7756

Citation: A. M. McDermott, S. Herberg, D. E. Mason, J. M. Collins, H. B. Pearson, J. H. Dawahare, R. Tang, A. N. Patwa, M. W. Grinstaff, D. J. Kelly, E. Alsberg, J. D. Boerckel, Recapitulating bone development through engineered mesenchymal condensations and mechanical cues for tissue regeneration. Sci. Transl. Med. 11, eaav7756 (2019). 


\section{Science Translational Medicine}

\section{Recapitulating bone development through engineered mesenchymal condensations and mechanical cues for tissue regeneration}

Anna M. McDermott, Samuel Herberg, Devon E. Mason, Joseph M. Collins, Hope B. Pearson, James H. Dawahare, Rui Tang, Amit N. Patwa, Mark W. Grinstaff, Daniel J. Kelly, Eben Alsberg and Joel D. Boerckel

Sci Transl Med 11, eaav7756.

DOI: 10.1126/scitransImed.aav7756

\section{Taking cues from development}

One way that bone forms during development is termed endochondral ossification, a process requiring a cartilage intermediate. McDermott et al. mimicked this process during bone defect repair in rats, implanting cylindrical constructs of human mesenchymal stem cells and transforming growth factor- $\beta 1$ into critical-sized femur defects and using fixation plates to apply mechanical loading. Loading altered cartilage persistence, blood vessel formation, and bone regeneration. Applying mechanical forces 4 weeks after construct implantation resulted in better bone bridging than loading at the time of implantation. This study demonstrates how mechanical loading can be used with tissue engineering to augment bone regeneration.

ARTICLE TOOLS

SUPPLEMENTARY MATERIALS

RELATED

CONTENT

REFERENCES

PERMISSIONS http://stm.sciencemag.org/content/11/495/eaav7756

http://stm.sciencemag.org/content/suppl/2019/06/03/11.495.eaav7756.DC1 http://stm.sciencemag.org/content/scitransmed/11/489/eaar4953.full http://stm.sciencemag.org/content/scitransmed/11/487/eaao0750.full http://stm.sciencemag.org/content/scitransmed/10/446/eaaq1802.full http://stm.sciencemag.org/content/scitransmed/3/100/100ra89.full http://stm.sciencemag.org/content/scitransmed/11/502/eaau7411.full http://stm.sciencemag.org/content/scitransmed/12/527/eaav7753.full

This article cites 74 articles, 9 of which you can access for free http://stm.sciencemag.org/content/11/495/eaav7756\#BIBL

http://www.sciencemag.org/help/reprints-and-permissions

Science Translational Medicine (ISSN 1946-6242) is published by the American Association for the Advancement of Science, 1200 New York Avenue NW, Washington, DC 20005. The title Science Translational Medicine is a registered trademark of AAAS.

Copyright @ 2019 The Authors, some rights reserved; exclusive licensee American Association for the Advancement of Science. No claim to original U.S. Government Works 